\title{
Cross-Industrial User Channel Preferences on the Path to Online Purchase: Homogeneous, Heterogeneous, or Mixed?
}

\author{
Ingo F. Becker, Marc Linzmajer \& Florian von Wangenheim
}

To cite this article: Ingo F. Becker, Marc Linzmajer \& Florian von Wangenheim (2017):

Cross-Industrial User Channel Preferences on the Path to Online Purchase: Homogeneous, Heterogeneous, or Mixed?, Journal of Advertising, DOI: 10.1080/00913367.2017.1300076

To link to this article: http://dx.doi.org/10.1080/00913367.2017.1300076

View supplementary material ¿

Published online: 27 Mar 2017.

Submit your article to this journal $₫$

View related articles $\smile$

View Crossmark data $\nearrow$ 


\title{
Cross-Industrial User Channel Preferences on the Path to Online Purchase: Homogeneous, Heterogeneous, or Mixed?
}

\author{
Ingo F. Becker \\ Swiss Federal Institute of Technology in Zurich, Zurich, Switzerland
}

Marc Linzmajer

University of St. Gallen, St. Gallen, Switzerland

Florian von Wangenheim

Swiss Federal Institute of Technology in Zurich, Zurich, Switzerland

\begin{abstract}
Though research literature addresses a broad range of advertising impact models, studies on the channel preferences of online purchasers have received little attention, regarding both multichannel settings and channel interplay in click sequences. To provide advertisers a method for better evaluating customer channel preference, this study investigates the path to purchase by building on four multichannel clickstream data sets from three industries, recorded with cookie-tracking technologies. Applying a Cox model and clustering techniques supports delineation of empirical generalizations and industry-specific findings on channel exposure, including their antecedents and distinct channel click sequences. Across data sets, online users show idiosyncratic channel preferences for a limited set of one or two channels rather than multiple online vehicles. Both channel homogeneous click sequences and combinations of two channels (including branded contacts) are effective as purchase predictors. Our study also presents industry-specific results regarding the influence of click sequences on purchase intent, thereby providing insights for advertising research, particularly as are suited to optimization of online advertising activities.
\end{abstract}

Online multichannel advertising refers to the practice of simultaneously offering customers information, goods, services, and support through a full range of synchronized channels. Managing firm-customer interactions in the context of

Address correspondence to Ingo F. Becker, Swiss Federal Institute of Technology in Zurich (ETH Zurich), Department of Management, Technology, and Economics (D-MTEC), Weinbergstr. 56/58, 8092 Zurich, Switzerland. E-mail: ingofbecker@web.de

Ingo F. Becker (PhD, ETH Zurich) is associated with ETH Zurich, D-MTEC, and chair of technology marketing, Zurich, Switzerland.

Marc Linzmajer ( $\mathrm{PhD}$, Zeppelin University) is a Postdoctoral Researcher and Head of the Competence Centre E-Commerce at the Institute of Retail Management, University of St. Gallen (IRM-HSG), St. Gallen, Switzerland.

Florian von Wangenheim (PhD, Johannes Gutenberg University) is a professor at ETH Zurich, D-MTEC, and chair of technology marketing, Zurich, Switzerland. integrated communication strategies and multichannel management—which includes determining the channel exposures (e.g., search, display) that precede a conversion-has become a cornerstone of online marketing strategy (Yadav and Pavlou 2014). Thus, developing a deeper understanding of online channel preferences, of the interplay of channels, and of the way that channels relate to customers' (underlying) browsing intentions has become increasingly important for managers and scholars alike.

Cross-channel research on direct marketing companies such as retailers and brick-and-mortar stores suggests that crosschannel advertising (including, for instance, online channels such as search engines, and offline channels such as print, radio, and television) constitutes synergetic cross-channel effects (e.g., spillover effects) that increase advertising effectiveness (Chang and Thorson 2004; Klapdor et al. 2015; Naik and Raman 2003; Wiesel, Pauwels, and Arts 2011). Research on offline and hybrid (i.e., online/offline) channels shows that multiple channels are perceived to be more credible and to increase processing motivation which, in turn, elevates brand recognition and purchase intent (MacInnis and Jaworski 1989).

However, it remains unclear whether the conclusions from cross-channel research generalize to a pure online environment (Li and Kannan 2014), which is typically classified along numerous channels. Online customers who use various channels while browsing (customers who prefer heterogeneous channels) provoke more interchannel spillover. But are these customers more likely to convert, thereby becoming more valuable than online customers who stay predominantly within one channel prior to purchase (i.e., customers who prefer homogeneous channels)? Yet established cross-channel knowledge may not apply, because in an offline or hybrid environment the media vehicles often differ significantly in their appearance and sensory stimuli (e.g., radio, print, television, laptop), while for online settings the hardware transporting media messages is more constant (e.g., the device's display). 
Existing research on online advertising effectiveness illuminates a number of events involving singular channels (Jerath, Ma, and Park 2014; Klapdor, von Wangenheim, and Schumann 2014; Rutz, Trusov, and Bucklin 2011) and proves that online channels affect one another (e.g., spillover effects)-though mostly in two-dimensional channel conditions (Rutz and Bucklin 2011; Yang and Ghose 2010). It follows that conclusions drawn from online research often refer to selected channels (or a limited set of channels) and thus do not provide a complete picture of the multichannel online environment (Braun and Moe 2013; Rutz and Bucklin 2011; $\mathrm{Xu}$, Duan, and Whinston 2014).

In the more comparative context of a full set of online channels, less information is available on present and past channel exposure, channel spillovers (heterogeneous channel interactions), and channel carryovers (homogeneous channel interactions), or the actual users' channel preferences along the path to purchase. To complement prior research in this multichannel e-commerce context, we address three consecutive research questions, focusing our interpretation on both generalizable and industry-specific findings:

RQ1: What channel contacts-including present channel clicks and past channel stock-indicate the formation of an online purchase decision?

RQ2: In the journey to purchase, are the customer's browsing preferences more channel homogeneous or channel heterogeneous?

RQ3: What specific channel sequences manifested in users' browsing traits-across industries and in industry-specific contexts-predispose purchase intent?

For the first two research questions, we apply a Cox proportional hazards model to analyze the full set of present and past channel clicks. Furthermore, by using interaction effects between present and past exposures, we examine the moderating role of prior homogeneous/heterogeneous channel exposure and present channel browsing (clicking) behavior, and investigate the link to customer conversions. For the third research question, we apply multiple cluster analyses to uncover specific channel click sequences consisting of up to four sequential clicks prior to the Cox model. Implementing these click sequences for the Cox model adds explanatory power to the explorative clustering technique. Building on four large-scale clickstream data sets from three different industries allows us to derive generalizable and industry-specific empirical conclusions.

Our research contributes innovative understandings to the literature of online advertising. First, we address channel effectiveness in a multichannel online context (Fulgoni and Mörn 2009; Klapdor et al. 2015). Contrary to previous understandings of cross-channel preferences (Naik and Raman 2003; Tellis et al. 2005), our results (for all data sets) show that customers in online environments who are likely to convert use one preferred channel (or a limited set of channels dominated by one specific channel). These results translate into user channel preferences on the path to purchase that are homogeneous rather than heterogeneous. Disentangling present from (repeated) past clicks reveals that present clicks on affiliates, branded paid and branded unpaid search, and social media, as well as (repeated) past clicks on retargeting, are positively associated with conversion events across data sets. Advertisers anticipating this behavior may create routes toward more valuable user segments.

Second, we translate exposures into specific online channel click sequences in a comparative, more complex manner (Danaher and Dagger 2013; Hui, Fader, and Bradlow 2009), leading to fine-grained, specific click patterns that support a better understanding of channel effectiveness (Ghose and Yang 2009; Rutz, Trusov, and Bucklin 2011). Across data sets and for four online companies, we confirm our prior claim for the consistent relevance of homogeneous channel sequences that mirror channel user preferences, a claim that is also indicated in multichannel relational communication (Godfrey, Seiders, and Voss 2011). It is useful for advertisers and retailers to recognize that the effect of homogeneous channel preferences remains comparably stable when the length of the analyzed click sequence is expanded up to four subsequent clicks. In contrast, for travel-related online searches, the effectiveness of specific channel homogeneous click sequences declines. Across data sets, though only selectively, both channel homogeneous click sequences and their channel heterogeneous equivalents have a stronger effect on conversion events. Still, the vast majority of these click sequences consist of no more than two channels, expressing the tendency of users to use a limited set of channels. While channel heterogeneous click sequences (including branded contacts) apply consistently across data sets, other contacts such as affiliates, referrers, price comparisons, and retargeted advertisements appear to be industry specific or driven by further underlying factors.

From a broader perspective, the more advanced understanding of online channel click sequences that results from our study generates meaningful insights into online user preferences, responding to calls for research that develops marketing impact models to maximize advertising impact based on individual-level customer path data (Hui, Fader, and Bradlow 2009). As well, our study reduces the gap between theory and practice (Little 2004), and adds practical cross-industry generalizations and industry-specific findings (Li and Kannan 2014).

\section{CONCEPTUAL DEVELOPMENT}

\section{Conceptual Model}

In recent years, analysis of clickstream data has advanced significantly. These advances can be grouped into three broad categories: (1) website use and navigation, (2) advertising on the Internet, and (3) online shopping and e-commerce. Therefore, many of the models have focused on whether a website visitor will complete a transaction and on the factors that may predict this conversion. By developing a literature review that 
details the impact of channel clicks and/or clickstreams on customer conversions (Table 1), we identify two basic research gaps. First, existing studies examine only a limited range of channels, which prevents advertisers from effectively allocating budget resources in the dynamic multichannel online environment. Second, we were not able to locate general results that address the impact of the entire clickstream on conversion across industries.

To fill these gaps in the literature, we use an explorative analysis approach. Based on the idea that time is a resource and, as such, consumers must make decisions regarding their use of time in the purchase of goods and services (LeClerc, Schmitt, and Dubé 1995), we hypothesize that customers use only a limited set of channel contacts on their path to purchase - that when faced with endless opportunities to gather information in the prepurchase phase, they can be impatient in the online environment. To identify a general clickstream pattern that better predicts customer conversions across industries, we applied the following conceptual model to all four industries analyzed in our study.

We first developed a conceptual model of the relationship between channel effects and conversions in order to analyze bipolar user positions, showing channel homogeneous browsing patterns on their path to purchase or channel heterogeneous browsing patterns (Model 1: general channel effects). The main model includes the effects of present and past channel exposures (clicks), and the interaction effects between present and past collective channel exposure (past stock). In addition, we added a base model that excludes the interaction effects (Figure 1).

For Model 1, we implemented the effect of present firm-customer contacts with the complete set of channels applied in our data sets. Setting the foundation for subsequent analysis of interaction effects, we captured the effectiveness of present channel contacts, also reflecting one-click journeys which do not include interactions but which account for a substantial share of customer journeys. Next, representing user browsing history, we captured the number of past channel contacts (past channel stock), separated into channel homogeneous variables and channel heterogeneous variables. Past channel exposure may affect future contacts and journey outcomes (Klapdor et al. 2015), which advertisers should take into account when they analyze current advertising performance indicators (Li and Kannan 2014).

To provide more detailed results, we further conceptualized specific channel effects, that is, the effect of specific channel click sequences that take place in user journeys toward conversion. As the number of potential channel click sequences increases exponentially in accord with the number of channels applied and the length of the click sequences modeled (the curse of dimensionality; Bellman 1961), examining certain effects (e.g., sequential click patterns) has inevitably required simplification. Hence, we applied a set of cluster analyses to uncover distinct browsing patterns within each data set. To ensure both a high level of detail and an adequate sample size, we implemented up to four sequential channel clicks within our cluster analyses, enabling our consecutive model. Consequently, in Model 2 we focused on specific channel sequences distilled from the preceding cluster analyses, measuring the effects of specific browsing patterns with two (Model 2a), three (Model 2b), and four sequential clicks (Model 2c). To alleviate potential multicollinearity, we refrained from modeling all effects within one model yet were able to retain unaffected cross-industry comparisons (Figure 1).

In Model 1, with a focus on general channel preferences, we implement interaction effects between present channel exposure and past homogeneous (and heterogeneous) channel exposure (past channel stock). While present channel effects omit the user's browsing history, aggregated past channel effects remain independent from subsequent (present) channel exposure. Thus, these interactions are well suited to extracting the effects of present channel exposure and their antecedents. The effects reflect the general user browsing pattern that covers the complete click history-for instance, to prefer channel homogeneous browsing paths rather than those that are channel heterogeneous. However, the exact channel click sequence remains hidden and, for instance, predominant channel homogeneous browsing paths may be interrupted be punctual clicks on alternative channels. Model 2 thus complements Model 1 by consolidating up to four specific channel click sequences into distinct variables, computing the effects of channel homogeneous as well as relevant channel heterogeneous click sequences. Applying these models to four data sets, we aim to identify cross-industry and industry-specific findings.

\section{The Four Data Sets}

We base our analyses on four sets of clickstream datarecords tracing the path individual users take while browsing the Internet - that were provided by online advertisers. We also worked in close cooperation with a multichannel tracking provider. Our technical data collection was based on cookie tracking, which delineates individual devices to approximate individual users. Cookie tracking serves as an invaluable input source for advertisers, allowing them to compile records of individual browsing histories, both from individual advertisers' websites and delivered by third-party advertising networks that track browsing activity across websites. Despite its value, however, tracking cookies has limitations. For instance, because cookie data track at the device level, multidevice use by a single user, or multiple users accessing one device, may be inaccurate. Users who delete cookies present another distortion of reported data (Flosi, Fulgoni, and Vollman 2013). Despite such limitations, cookie-generated data remain a widely accepted standard for industry and research (Tucker 2013).

Cookies provide specific useful information for our data sets. We are able to document each visit to an advertiser's website with the origin of the click (either a direct type-in search using the advertiser's universal resource locator (URL) or a user click on an advertising message), a time stamp accurate to the 


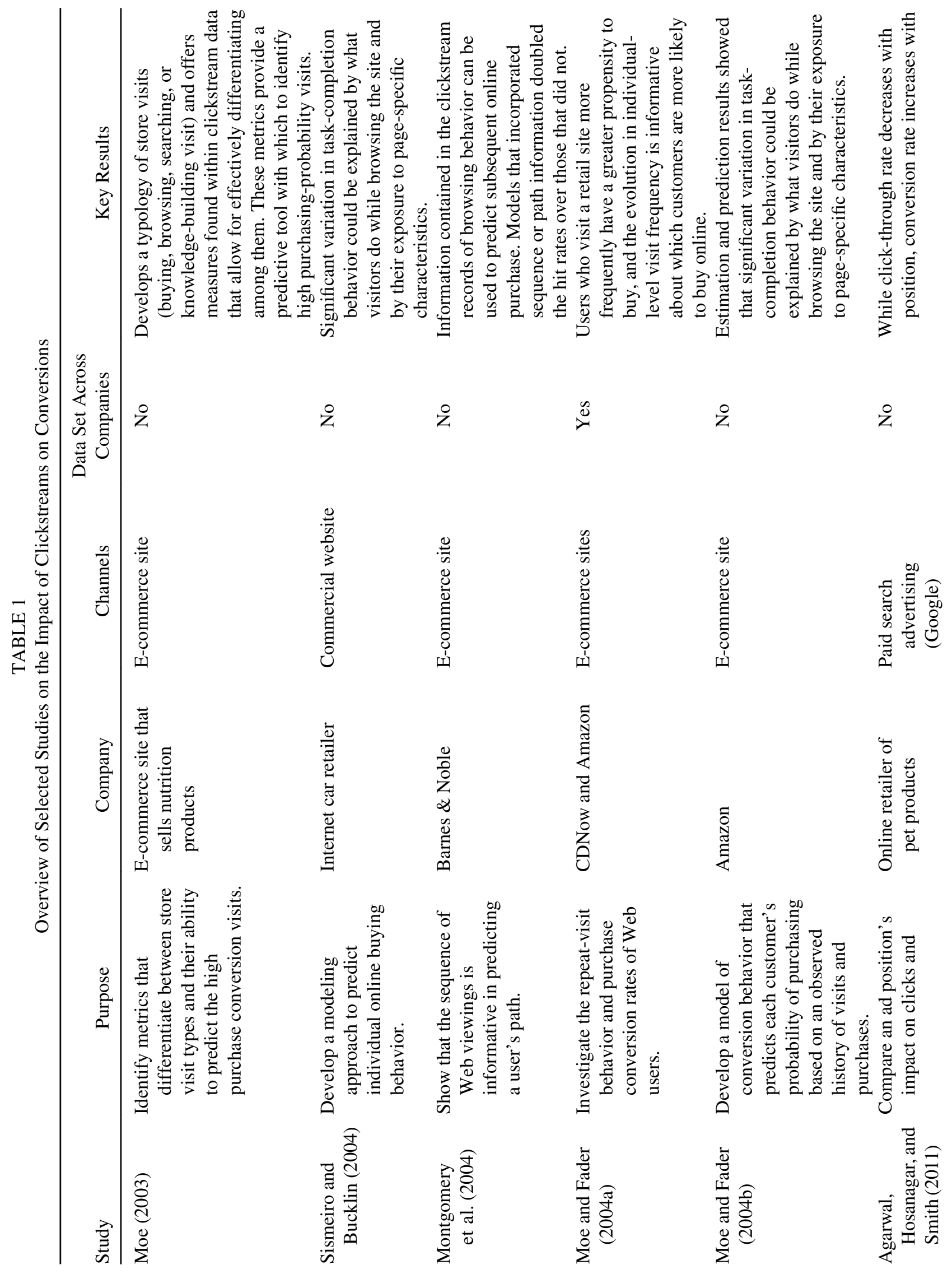



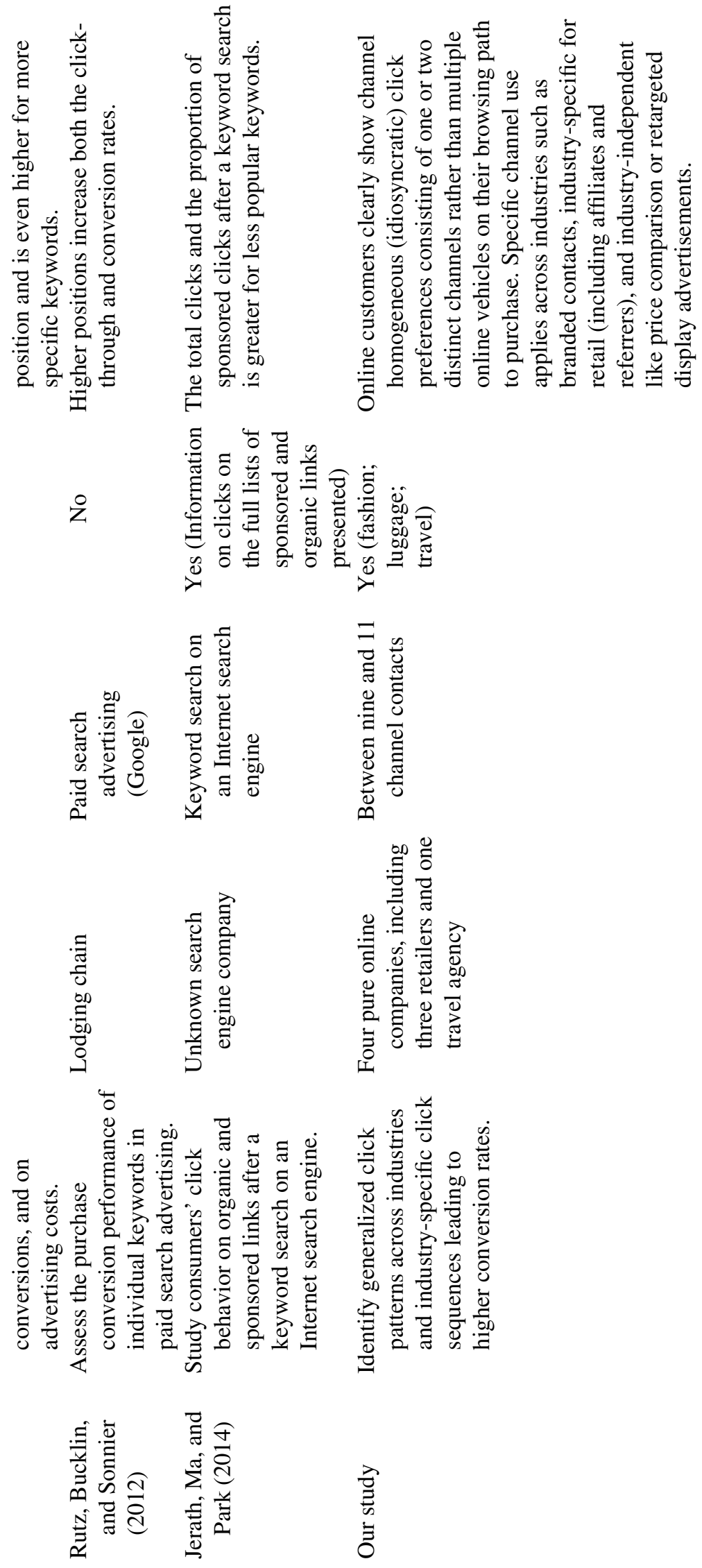

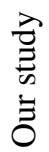




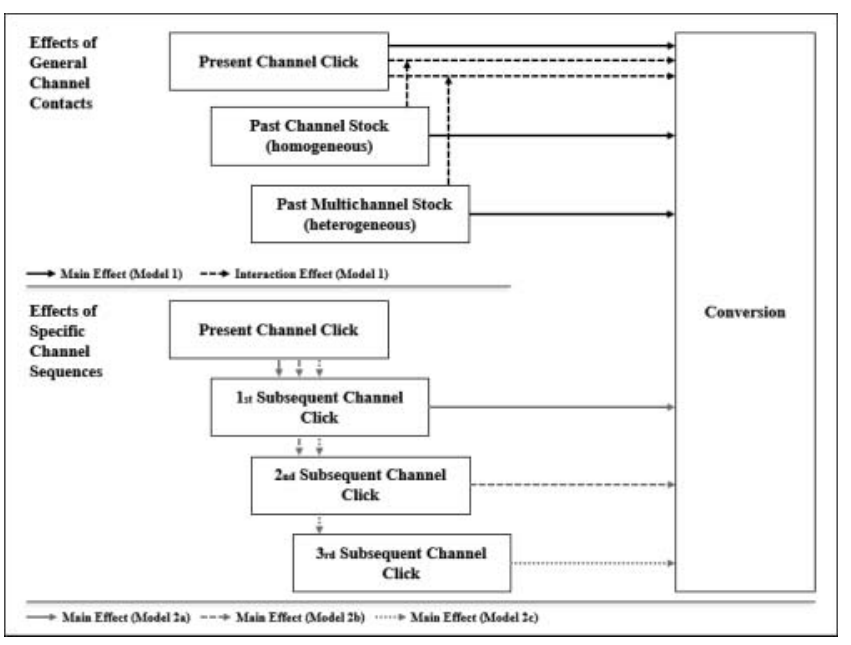

FIG. 1. Conceptual model of relationships between channels, channel sequences, and conversions.

second, and a unique user identification (ID) associated with an individual device. Particularly useful for our research are the cookie data that indicate whether a site visit was followed by a conversion event within 30 days, or whether it was a nonconverting journey. We consolidated the data records for each advertiser into one data set, maintaining unique user IDs to allow for analysis at the most granular level.

We selected pure online players as data providers, facilitating exclusion of online/offline cross-channel effects. These advertisers operate in three different industries, namely, two online fashion stores (high priced and midlevel), one online luggage retailer, and one online travel agency. Selecting fashion retailers with different price ranges allows us to make within-industry comparisons yet with different price sensitivity of users. The luggage retailer complements retail-specific comparisons, whereas results from the travel agency support analysis of differences and commonalities between retail and service companies. We further controlled for tracking errors, such as double-counts of identical clicks, taking into account the corresponding channel information and time stamp, as well as nonhuman click behavior caused by web bots. Overall, we excluded approximately $2.5 \%$ of all clicks. Table 2 presents a detailed overview of the data sets. Detailed definitions of the online channels appear in the appendix.

In our data sets, search engine advertising (SEA) signifies paid ads on Google's search engine, and search engine

TABLE 2

Descriptive Statistics of the Data Sets, Including Channels

\begin{tabular}{lcccc}
\hline Industry & $\begin{array}{c}\text { Data Set 1 } \\
\text { (Fashion, High Priced) }\end{array}$ & $\begin{array}{c}\text { Data Set 2 } \\
\text { (Fashion, Midlevel) }\end{array}$ & $\begin{array}{c}\text { Data Set 3 } \\
\text { (Retail Luggage) }\end{array}$ & $\begin{array}{c}\text { Data Set 4 } \\
\text { (Travel) }\end{array}$ \\
\hline Number of different channels & 11 & 11 & 10 & 9 \\
Number of different channels analyzed & 10 & 8 & 9 & 9 \\
Number of journeys & $1,184,582$ & 862,114 & 405,343 & 600,872 \\
Journey length in clicks & $1.38(1.88)$ & $1.30(1.23)$ & $1.48(1.28)$ & $2.30(5.20)$ \\
Number of different channels per journey & $1.06(0.32)$ & $1.06(0.27)$ & $1.09(0.33)$ & $1.10(0.33)$ \\
Number of conversions & 10,153 & 16,201 & 8,117 & 9,861 \\
Journey conversion rate & $0.86 \%$ & $1.88 \%$ & $2.00 \%$ & $1.64 \%$ \\
Number of clicks & $1,635,724$ & $1,122,838$ & 601,417 & $1,380,190$ \\
Affiliate & 754,355 & 2 & 1,699 & 36,487 \\
Display & 10 & - & - & 787,743 \\
Newsletter & 79,453 & 12 & 8 & 17,204 \\
Price comparison & 2,205 & - & 15,003 & 90,302 \\
Referrer & 70,394 & 123,952 & 15,043 & - \\
Retargeting & 20,059 & 31 & 7,011 & 5,697 \\
Paid search generic & 120,542 & 119,374 & 442,617 & 287,346 \\
Paid search branded & 50,982 & 37,493 & 8,284 & 54,481 \\
Unpaid search generic & 123,850 & 330,081 & 62,179 & 73,492 \\
Unpaid search branded & 75,030 & 42,380 & 3,866 & 27,438 \\
Direct type-in & 338,844 & 213,053 & 45,707 & - \\
Social media & - & 241,462 & - & - \\
Other & - & 14,998 & - & - \\
\hline
\end{tabular}

Note. Standard deviations are in parentheses. The different numbers of channels by advertiser are derived from the individual channel propensity and the selection of each advertiser. Rare channels are removed from the models, as they are underrepresented and insignificant. DS 1 = display; DS 2 = affiliate, newsletter, retargeting; DS 3 = newsletter). 
optimization (SEO) refers to an unpaid organic search on Google. Both appear in all four data sets and are separated into different channels for branded and generic search terms, because they reflect different user browsing states (Rutz and Bucklin 2011). The frequency of channel exposure varies considerably across the four data sets, alleviating endogeneity concerns. To further rule out potential endogeneity, we ran models that excluded retargeting and newsletter advertising, as these may interrelate with previous contacts.

\section{MODEL DEVELOPMENT}

\section{General Model Formulation}

The aim of our customer channel preference analyses is twofold. First, we focus on general channel effects on conversion events, including individual channel clicks and channel interactions, to examine the effects of homogeneous/heterogeneous channel usage along the full journey (Model 1). Second, we deepen our general analysis of homogeneous/heterogeneous channel preferences by investigating distinct effects of specific channel click sequences on conversion events (Model 2).

To account for right-censoring and to reflect the sequential nature of the path data, we applied a Cox proportional hazards model (Cox 1972). The dependent variable is time $t$ leading up to the occurrence of a binary event (here, a purchase event). The model formula describes the hazard at time $t$ as the product of two quantities-first, the baseline hazard, $\mathrm{h}_{0}(t)$, which defines the hazard per time unit $t$ at the baseline of the covariates and, second, the exponential expression $e$ to the linear sum of $\beta_{i} X_{i}$, with the sum over $p$ predictor $X$ covariates, which defines the responsive effect of the predictor covariates on the hazard. The covariates are modeled time independently, as our tests suggest the proportional hazards assumption holds. Being unaware of the particular form of the underlying hazard, we applied a semiparametric Cox model (Cox 1972), which derives the hazard as multiplicative replica directly from the data, increasing flexibility. The Cox model $h_{i}(t, X)$ is defined as

$$
h_{i}(t, X)=h_{0}(t) \times \exp \left(\sum_{j=1}^{p} \beta_{j} X_{i j}\right),
$$

with $X=\left(X_{1}, X_{2}, \ldots X_{p}\right)$ predictor variables for customer $i$. Following prior research to untangle data to the most disaggregated units (Tellis and Franses 2006), we based our models on individual customer journeys and we aggregate the time $t$ over days (Lambrecht and Tucker 2013).

\section{Modeling General Channel Contacts (Model 1)}

Main effects. Although we consistently apply a Cox proportional hazards model to investigate channel effects, the preparation and setup of Model 1 and Model 2 follow specific features. Consequently, the vector of covariates in our base Model 1 for user $i$ is specified as follows:

$$
\begin{aligned}
\exp \left(\sum_{j=1}^{p} \beta X_{i j}\right)= & \exp \left(\beta X_{i 1}+\beta X_{i 2}+\cdots+\beta X_{i j}+\beta \text { Past } X_{i 1}\right. \\
& +\beta \text { Past } X_{i 2}+\cdots+\beta \text { Past } X_{i j}+\beta \text { Channel } X_{\mathrm{i} 1} \\
& \left.+\beta \text { Channel } X_{i 2}+\cdots+\beta \text { Channel } X_{\mathrm{ij}}\right)
\end{aligned}
$$

While the baseline hazard $h_{0}(t)$ captures the time effect, aggregated over days, the vector of covariates captures the effect of present channel exposures, past homogeneous channel exposures, and past heterogeneous (multi)channel exposures. In particular, $X_{i j}$ signifies present clicks on a specific channel $\mathrm{j}$. Furthermore, Past $X_{i j}$ corresponds to the total number of clicks on channel $j$ before each present click, thus collecting preceding homogeneous channel exposures (i.e., idiosyncratic channel preferences). Similarly, Channel $X_{i j}$, captures the number of different channels the user is exposed to before each present click, excluding the channel of the present click. In other words, the covariate Channel $X_{i j}$ captures the number of heterogeneous (multi)channel occurrences prior to a click on channel $j$ apart from channel $j$-and thus excluding homogeneous channel exposures. An important feature of this definition is that we model past channel exposures by their exact occurrence, including past exposures both before and on the same day, a method unique to our study, as prior research includes only exposures prior to the corresponding day (e.g., Lambrecht and Tucker 2013).

Interaction effects. To better understand user channel preferences, we extend the base model by adding interaction effects. While the base model includes predictors for present and past channel exposures, the vector of covariates in the main Model 1 further captures interaction effects between present channel exposures and accumulated past channel exposures-including both preceding homogeneous and heterogeneous channel contacts. In this way we measure the effects of idiosyncratic and multichannel user preferences on their path to purchase:

$$
\begin{aligned}
\exp \left(\sum_{j=1}^{p} \beta \mathrm{X}_{\mathrm{ij}}\right)= & \exp \left(\beta \mathrm{X}_{\mathrm{i} 1}+\cdots+\beta \mathrm{X}_{\mathrm{ij}}+\beta \text { Past }_{\mathrm{i} 1}+\cdots\right. \\
& +\beta \text { Past }_{\mathrm{ij}}+\beta \text { ChannelX }_{\mathrm{i} 1}+\cdots \\
& +\beta \text { ChannelX }_{\mathrm{ij}}+\beta \mathrm{X}_{\mathrm{i} 1} \times \beta \text { Past }_{\mathrm{i} 1}+\cdots \\
& +\beta \mathrm{X}_{\mathrm{ij}} \times \beta \text { Past }_{\mathrm{ij}}+\beta \mathrm{X}_{\mathrm{i} 1} \\
& \left.\times \beta \text { ChannelX }_{\mathrm{i} 1}+\cdots+\beta \mathrm{X}_{\mathrm{ij}} \times \beta \text { ChannelX }_{\mathrm{ij}}\right) .
\end{aligned}
$$




\section{Modeling Specific Channel Sequences (Model 2)}

Defining the covariates. A related challenge to investigating channel preferences is associated with the rise of channel diversity and journey length, which further provokes a phenomenon known as the "curse of dimensionality" (Bellman 1961). To encounter this phenomenon, we proposed cluster analysis as the data-mining technique to uncover relevant variables prior to application of the Cox model. For each data set, we conducted four cluster analyses, building on the full sample by including one-click journeys, as well as reducing samples that comprise journeys with at least two, three, and four clicks. Thereby, we ensured that we would identify relevant click patterns that encompass more than one click. To determine the number of clusters, we relied on the variance ratio criterion (VRC) (Calinski and Harabasz 1974), as it is considered one of the best-suited criteria (Milligan and Cooper 1985). Accordingly, we computed the VRC for a range of cluster solutions that leverage the $\omega$ statistic to identify the cluster number that best fits the data (Mooi and Sarstedt 2011). For each data set and cluster analysis, we compared cluster solutions ranging (Hubert and Arabie 1985) from two to 25 clusters to identify the most adequate number of clusters. To ensure consistency, we harmonized the number of cluster solutions for each sample that was suitable across all data sets. To form distinct clusters, we applied widely accepted $K$-means clustering initialized with centroids derived from Ward's (1963) hierarchical clustering approach, using squared Euclidean distances (Hubert and Arabie 1985; Steinley and Brusco 2007). Finally, we translated the retrieved cluster centers into sequential click patterns that may serve as focused input variables in our consecutive Cox models on the effects of specific channel click patterns. Thus, we approached the dichotomy between the curse of dimensionality (Bellman 1961) and distilling relevant patterns within the richness of data.

Preparing the model. Building on the cluster analyses, we developed individual models to investigate specific click patterns, including two (Model 2a), three (Model 2b), and four sequential clicks (Model 2c). As click patterns consisting of two consecutive clicks may overlap click patterns of, for instance, three clicks, to rule out (multi)collinearity we refrained from implementing all covariates into one model. In addition to channel heterogeneous click sequences, channel homogeneous click sequences appear to be prevalent across all data sets and clustering approaches, specifying the vector of covariates for user $i$ as follows (Model 2a):

$$
\begin{aligned}
\exp \left(\sum_{j=1}^{p} \beta X_{i j}\right)= & \exp \left(\beta\left(X_{i 1}-X_{i 1}\right)+\cdots+\beta\left(X_{i j}-X_{i j}\right)\right. \\
& \left.+\beta\left(X_{i a}-X_{i b}\right)+\cdots+\beta\left(X_{i i}-X_{i j}\right)\right)
\end{aligned}
$$

The covariates measure two different types of effects covering homogeneous and heterogeneous click sequences.
Specifically, $\left(X_{i j}-X_{i j}\right)$ measures the effect of channel homogeneous click sequences for user $i$ and channel $j$, while $\left(X_{i i}-X_{i j}\right)$ measures the effect of channel heterogeneous click sequences for user $i$ and channels $i$ and $j$. It is noteworthy that the latter effect correctly reflects the chronological order of each click sequence and applies only for covariates derived by the cluster analyses. Model 2b and Model 2c are specified analogously, reflecting click sequences of three and four clicks.

\section{ESTIMATION RESULTS}

We concentrate our interpretation on main Model 1, as it also covers interaction effects as well as all variants of Model 2 . The interpretation is contextual; the data sets do not include information on users who did not interact with the focal advertiser's website. Accordingly, a negative coefficient does not necessarily imply that the corresponding clicking behavior of a user represents a lower purchase propensity than someone who never visited the website. Also, the magnitude of a particular effect is not fully comparable between data sets, as all data sets are unique insofar as they may comprise different channels, channel fractions, and creative content. Yet the magnitude of effects within each model provides a clear direction of the click behavior indicating purchase intent.

\section{Effects of General Channel Contacts (Model 1)}

Present channel effects. The effects of website visits are consistently positive across data sets, from present website visits, through affiliates, social media, branded paid and branded unpaid search, and direct type-ins. In contrast, the effects of both present clicks on display advertisements and referrers tend to be negative. For the remaining channels-newsletters, price comparisons, retargeting, generic paid and generic unpaid search - the picture is not clear-cut across data sets (Table 3).

Affiliates serve as a gateway to a website. Because users already anticipate where they are being transferred, users who have a definite idea about their purchase intent confront the potential to be encouraged to use an affiliate contact on their path to purchase (DS 1: Affiliate $b=0.061, p<.01$; DS 3: Affiliate $b=0.285, p<.01$; DS 4: Affiliate $b=0.200, p<$ .01). In social media, for instance, the user who is redirected to an advertiser's offering must leave the current activity. However, if a user decides to leave a current web location to follow an advertisement, the ad must have raised strong interest or indicated a useful response to a need, indicating that social media clicks may be a relevant source for anticipating conversion events (DS 2: Social $b=0.092, p<.01$ ). Direct type-ins, in contrast, involve only those users who are already aware of the existence of a corresponding website and who, at least in part, know what that website offers. Thus, more directed (navigational) users may apply such a channel (DS 1: TypeIn $b=$ $0.126, p<.01$; DS 2: TypeIn $b=0.061, p<.1)$. The same 


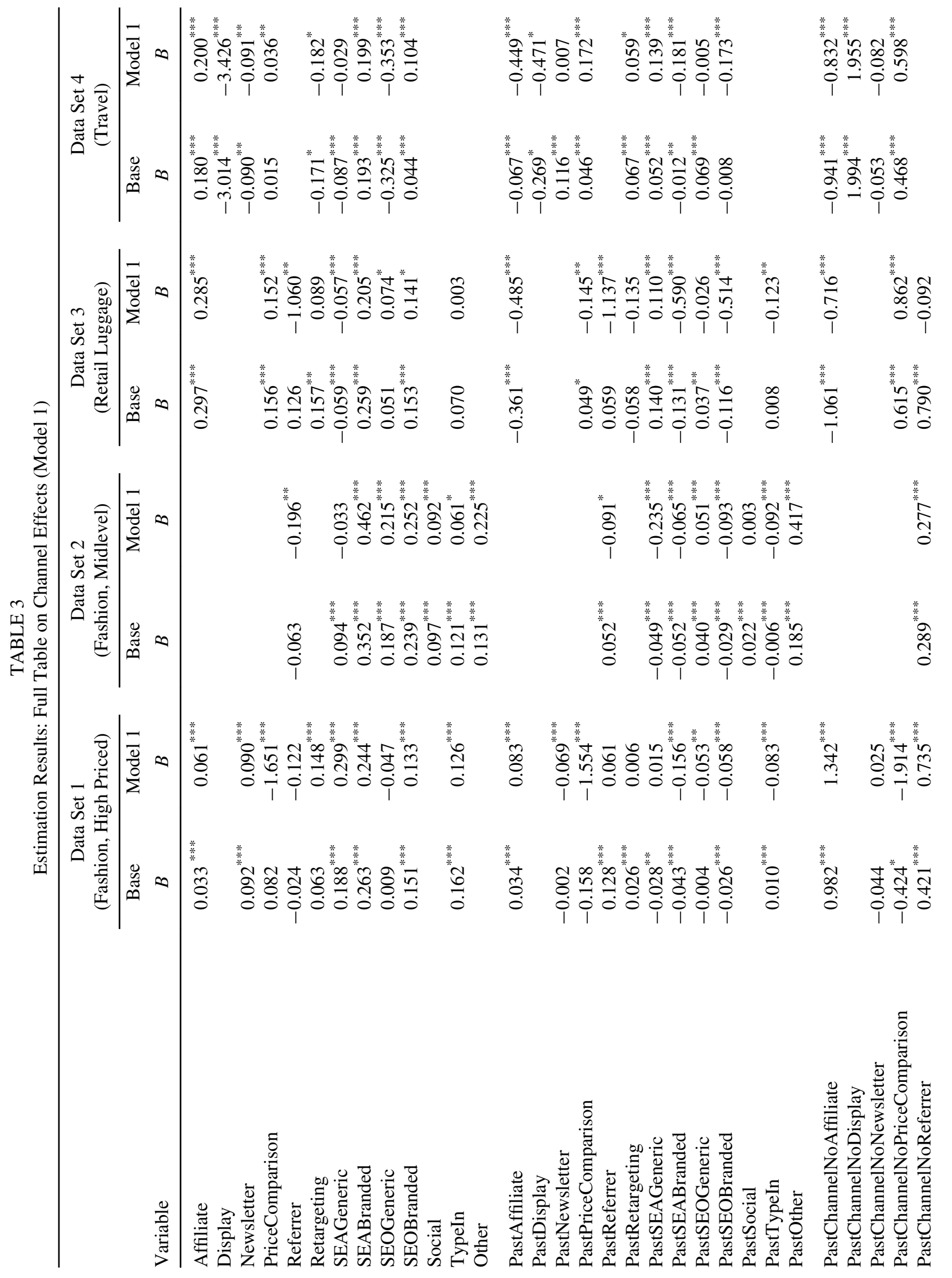




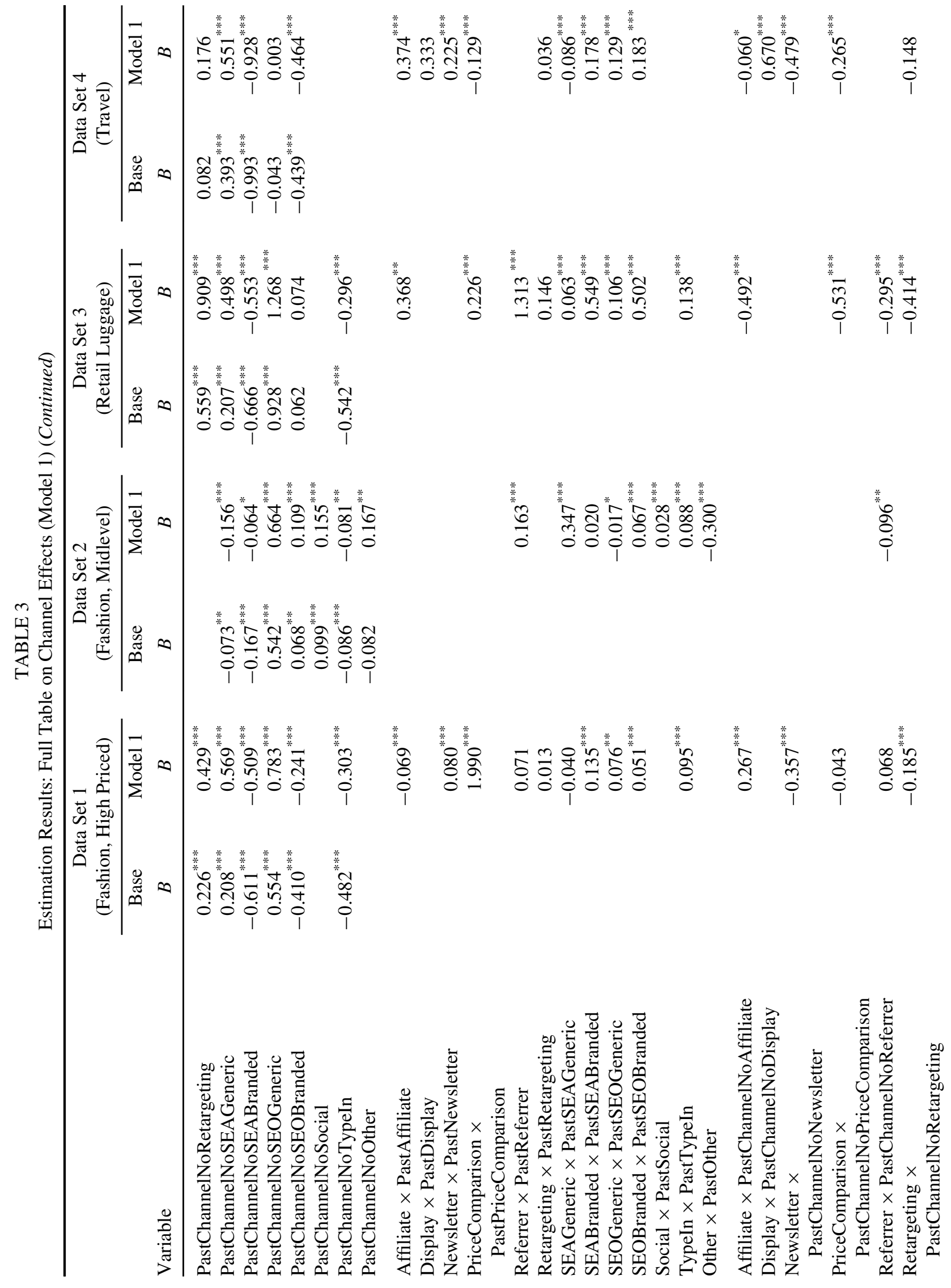




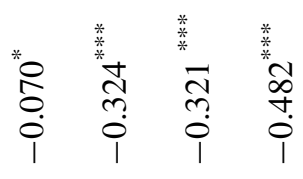

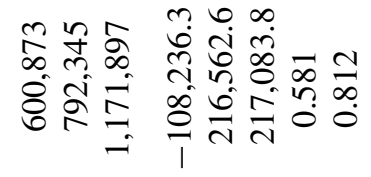

m ๘

के

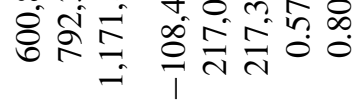

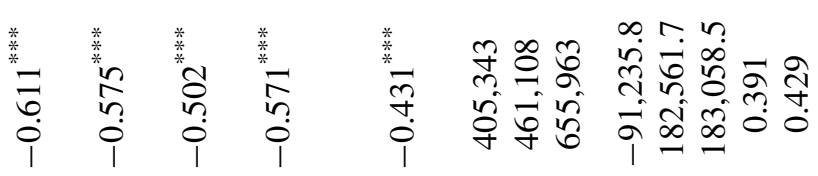

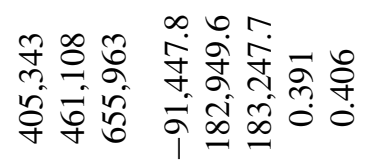

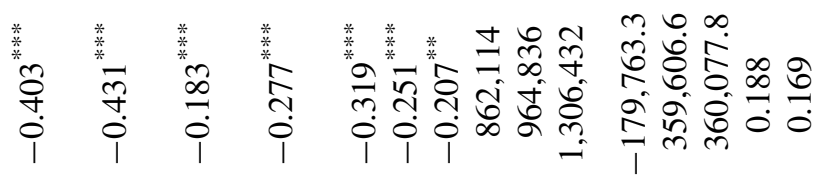

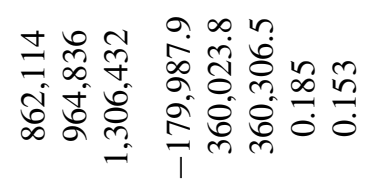

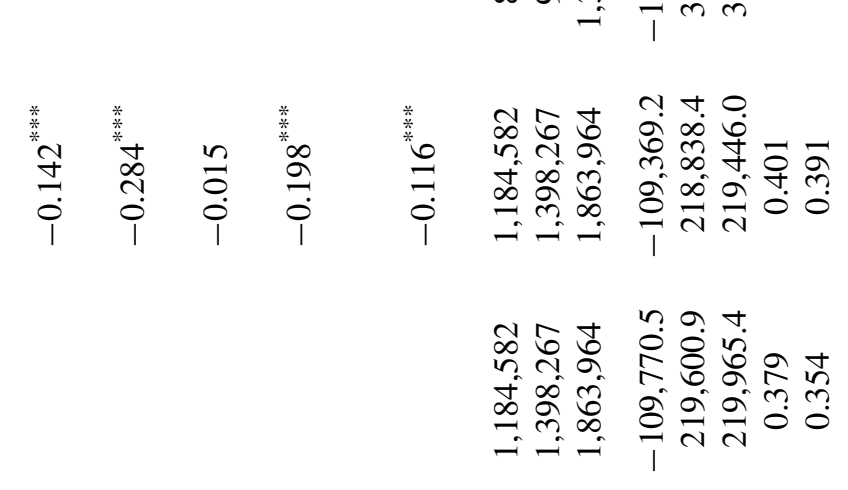

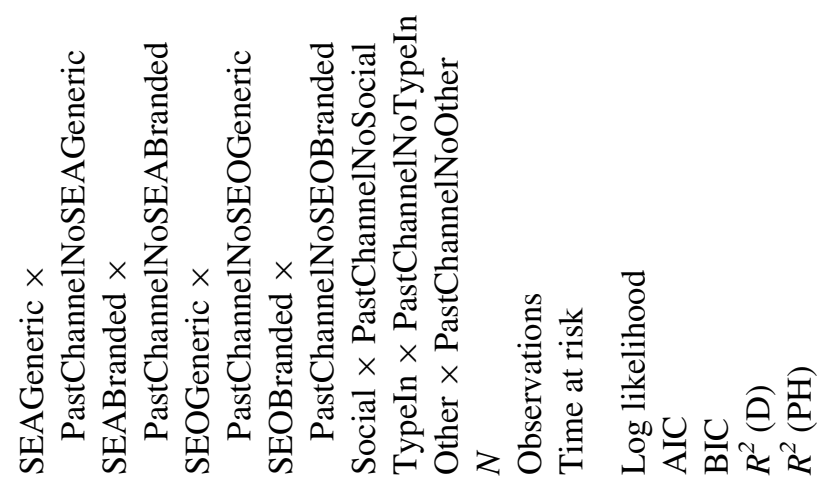

$\overline{0}$

$V$

**

$\ddot{8}$

ह

:

$\stackrel{8}{\Xi}$

i $v$ 
logic may hold for branded search, including paid search (e.g., DS 1: SEAbranded $b=0.244, p<.01$; DS 2: SEAbranded $b=0.462, p<.01)$ and unpaid search at a somewhat lower magnitude (e.g., DS 1: SEObranded $b=0.133, p<.01$; DS 2: SEObranded $b=0.252, p<.01)$. Interestingly, different rules seem to apply for a generic search. In our data sets, generic search clicks show mostly positive effects for online retailers (e.g., DS 1: SEAgeneric $b=0.299, p<.01$; DS 2: SEOgeneric $b=0.215, p<.01)$, but they reveal negative effects for the travel website (DS 4: SEOgeneric $b=-0.353, p<.01$ ). Prior research on paid search concludes that generic paid search has higher apparent costs compared to branded paid search, thereby creating spillovers to subsequent branded search (Jansen, Sobel, and Zhang 2011; Rutz and Bucklin 2011). While our results indicate congruence with the first claim, in Model 1 we do not specifically focus on interactions between generic and branded search, which may further enhance the advertising value of a generic search. Notwithstanding these interactions, a branded search is more directed (navigational), while a generic search is broader (informational), also indicating a different user state within the purchase deliberation process (Jansen, Sobel, and Zhang 2011; Klapdor et al. 2015). A newsletter indicates positive effects for fashion retail yet negative effects for travel (DS 1: Newsletter $b=0.090, p<.01$; DS 4: Newsletter $b=-0.091, p<.05)$. This deviation may be explained by the dissimilar nature of the two product categories. While fashion newsletters may attract users to take advantage of a time-limited offering (e.g., sale, discount campaign), the acceptance of a travel offering requires more complex preconditions, for example, leaving work for a holiday period, or group decision making. Therefore, users reacting to newsletters may simply be curious when viewing the offering or its price, not knowing their actual ability to accept a special offer. A similar explanation may apply for a retargeted display. While a retargeted display may reawaken interest in retail products, travel products are subject to more external influences (DS 1: Retargeting $b=0.148, p<.01$; DS 3: Retargeting $b=0.157, p<.05$; DS 4: Newsletter $b=-0.182, p<.1$ ). Moreover, the coefficients for price comparison websites show deviating signs (DS 1: PriceComparison $\mathrm{b}=-1.651, p<.01$; DS 2: PriceComparison $b=0.156, p<.01$; DS 4: PriceComparison $b=0.036, p<.05)$. Accordingly, price competitiveness and degree of product commoditization may influence user choice (Mehta, Rajiv, and Srinivasan 2003). With regard to negative effects, display advertising, as it is initiated by the advertiser, may increase user awareness or interest (Chatterjee, Hoffman, and Novak 2003) or may affect changes in future purchase intent (Braun and Moe 2013; Fulgoni and Mörn 2009). In the relative context of our study, however, and with regard to immediate effects, our results suggest that display advertising is negatively associated with purchase propensity (DS 4: Display $b=-3.426, p<.01$ ). Still, display exhibits positive effects-on search, for instance-leading to an overall increase of return on investment (ROI) (Kireyev, Pauwels, and Gupta 2013). That is why display advertising may be worth investing in for the longer term, as its effects may last for a comparatively longer period (Breuer, Brettel, and Engelen 2011). Referral campaigns comprise the second consistently negative channel effect across data sets (DS 2: Referrer $b=-0.196, p<.05$; DS 3: Referrer $b=-1.06, p<.05)$. The relationship between the referrers and the referred user is closer than in affiliate marketing, in the sense that the referrer website is not necessarily compensated in a remuneration model. Thus, because it is not part of the referrer's business model, the desire to refer a customer to a web shop may be less pronounced.

Past channel effects. Next, we concentrate on the repeated use of particular channels during the browsing history. The past use of retargeting shows positive effects on conversions, while branded search as well as direct type-ins are negatively associated with conversions. The remaining channels show results that are more diverse across the data sets.

With regard to retargeting, the coefficients reveal a slightly positive effect (DS 4: PastRetargeting $b=0.059, p<.1$ ), which is especially interesting for advertisers who are concerned about frequency capping in the context of retargeted display advertisements. Thus, higher repetition rates of retargeted banners may reactivate users on their path to purchase. In contrast, for search, branded search especially (both paid and unpaid) - a repeated use of branded keywords—indicates that users are either indecisive or, over time, are losing their purchase interest in a specific product offered by a web shop (e.g., DS 1: PastSEAbranded $b=-0.156, p<.01$; DS 2: PastSEAbranded $b=-0.065, p<.01$; DS 3: PastSEAbranded $b=-0.590, p<.01$; DS 4: PastSEAbranded $b=-0.181$, $p<.01)$. Direct type-ins show similar browsing and purchasing patterns (DS 1: TypeIn $b=-0.083, p<.01 ;$ DS 2: TypeIn $b=-0.092, p<.01$; DS 3: TypeIn $b=-0.123, p<.05$ ). Both branded search and direct type-ins are perceived to be navigational channels with a higher degree of contextual matching, indicating that the user already has a (pre)defined idea of what to expect (Broder 2002). As present contacts via the corresponding channels positively influence the time to conversion, and numerous past equivalent contacts are negative, the user decides to conclude with a purchase event in the short term (after the contact point) or, alternatively, may be unlikely to purchase at all. The results for generic search (paid and unpaid) are mixed and depend on the data set (e.g., DS 2: PastSEAgeneric $b=-0.235, p<.01$; DS 3: PastSEAgeneric $b=0.110, p<.01$; DS 4: PastSEAgeneric $b=0.139, p<$ $.01)$. These contacts indicate the informational nature of the browsing stage, which, in some cases, may indicate information acquisition and, in consequence, may still result in purchase transactions (e.g., DS 2: PastSEOgeneric $b=0.051, p<$ $.01)$. Although the negative effects slightly outweigh the positive, affiliates, the effects of referrers, price comparison, and newsletter should be specifically analyzed for each advertiser. Hereby, repeated contacts seem to indicate interest, though do 
not necessarily direct users toward purchase events. For instance, a customer who frequently uses an affiliate seems indecisive; otherwise, a purchase transaction will instead follow on short response (DS 1: PastAffiliate $b=0.083, p<.01$; DS 3: PastAffiliate $b=-0.485, p<.01$; DS 4: PastAffiliate $b=-0.449, p<.01$; e.g., DS 3: Affiliate $b=0.285, p<.01$ ).

Across data sets, the results for past repeated contacts are more ambiguous, as the results for present contacts limit generalized recommendations and increase the necessity for analyzing these effects on a case-by-case level. Their joint consideration may help in defining rules for frequency capping in real-time bidding applications.

Homogeneous and heterogeneous channel interactions. Prior offline and hybrid research has proved that multiple channels prompt sales synergies (e.g., Chang and Thorson 2004). Extending this effect to an online context, we analyze whether users exhibit dedicated homogeneous or heterogeneous channel preferences.

Our study results reveal a surprisingly clear picture across all data sets. The effects of interchannel, homogeneous spillovers become mostly significant and positive (e.g., DS 1: SEAbranded $\times$ PastSEAbranded $b=0.135, p<.01$; DS 2: SEAgeneric $\times$ PastSEAgeneric $b=0.347, p<.01$; DS 3: TypeIn $\times$ PastTypeIn $b=0.138, p<.01$; DS 4: Affiliate $\times$ PastAffiliate $b=0.374, p<.01)$. The heterogeneous channel interaction effects that have prior multichannel exposure become mostly significant and negative (e.g., DS 1: SEAbranded $\times$ PastChannelsNoSEAbranded $b=-0.284$, $p<.01$; DS 2: SEAgeneric $\times$ PastChannelsNoSEAgeneric $b=-0.403, p<.01$; DS 3: TypeIn $\times$ PastChannelsNoTypeIn $b=-0.431, p<.01$; DS 4: Affiliate $\times$ PastChannelsNoAffiliate $b=-0.060, p<.05)$. In other words, users seem to prefer online channels on their path to purchase. Multiple channel exposures, manifested in heterogeneous channel utilization, are perceived as interruptive, and thus are associated with a lower relative purchase propensity (Xia and Sudharshan 2002).

Although our findings suggest idiosyncratic channel preferences, on a more detailed level punctual exceptions may apply. With regard to homogeneous channel interactions, the coefficients for affiliates (DS 1: Affiliate $\times$ PastAffiliate $b=-0.069, p<.01$ ), price comparison (DS 4: PriceComparison $\times$ PastPriceComparison $b=-0.129, p<.01)$, generic paid search (DS 4: SEAgeneric $\times$ PastSEAgeneric $b=-0.086, p<.01$ ), and generic unpaid search (DS 2: SEOgeneric $\times$ PastSEOgeneric $b=-0.017, p<.10$ ) become significant and show a (slightly) negative sign. From the perspective of present and past click exposure equivalents, all these negative effects are associated with significant and positive effects (e.g., DS 1: Affiliate $b=0.061, p<.01$; DS 1: PastAffiliate $b=0.083, p<.01$ ). Obviously, these channels may exhibit negative repetitive effects. Yet these effects are statistically positive indicators, particularly if they are treated as decoupled from their channel pendants (present exposure) or if their channel descendant is left undefined (past exposure).
These results demonstrate that findings in advertising effectiveness research may not necessarily be generalized from a single data set. With regard to heterogeneous channel preferences, our four data sets show two interesting exceptions. First, the interaction between present affiliate clicks and the number of prior channels shows a significant and positive coefficient (DS 1: Affiliate $\times$ PastChannelsNoAffiliate $b=0.267, p<$ .01). In some cases the affiliate seems to be fertilized by the use of diverse channels. Given that affiliates may include review or coupon websites, users who include affiliates may be more advanced in Internet channel usage (Lambrecht and Tucker 2013). They may, therefore, use multiple channels to seek specific deals, culminating in an affiliate visit before concluding a purchase event. Second, with regard to the travel company, display advertising is stimulated by prior multichannel use (DS 4: Display $\times$ PastChannelsNoDisplay $b=0.670$, $p<.01$ ). From our previous analysis, we know that present and cumulated past display exposure measured in isolation shows negative effects. In contrast to other channels, a display may not exhibit a self-amplifying effect through repeated use. Instead, display users who (actively) visit a corresponding website via multifaceted paths appear to be more responsive to firm-initiated media, such as display advertising (Shankar and Malthouse 2007). The direction of the interchannel stimulation is remarkable, as prior research suggests that display may fuel indirect effects such as brand awareness and direct purchase intent (Qiu and Malthouse 2009). Prior research has also shown synergetic effects, such that display may raise search conversion and related metrics (Kireyev, Pauwels, and Gupta 2013). While we may not refute the existence of these effects, we demonstrate counterdirectional effects, from multiple channel exposures, toward display. As both exceptional effects occur within one unique data set, we may not generalize these findings but instead recommend an analytical evaluation on a case-by-case foundation.

\section{Effects of Specific Channel Sequences (Model 2)}

While Model 1 covers present channel clicks and reveals that users who are prone to convert show idiosyncratic channel preferences rather than multichannel preferences throughout the full journey, it may not completely capture the exact sequential nature of browsing patterns, as - based on Model 1-a user predominantly browsing via one channel may still have clicked on more than one channel on the path to purchase. To verify our first findings and to further elucidate exact browsing patterns, we turn to Model 2, which is delineated into three models to investigate click sequences of two clicks (Model 2a; see Table 4), three clicks (Model 2b; see Table 5), and four clicks (Model 2c; see Table 6).

Cluster analysis. In addition to identifying relevant input variables for Model 2, a glance at uncovered browsing pattern shows at least three interesting insights (see Table 7 to Table 22 in the appendix section). First, looking at cluster solutions 
TABLE 4

TABLE 4. Estimation Results: Full Table on Browsing Patterns With Two Clicks (Model 2a)

\begin{tabular}{|c|c|c|c|c|}
\hline Variable & $\begin{array}{c}\text { Data Set } 1 \\
\text { (Fashion High Priced) } \\
B\end{array}$ & $\begin{array}{c}\text { Data Set } 2 \\
\text { (Fashion Midlevel) } \\
B\end{array}$ & $\begin{array}{c}\text { Data Set } 3 \\
\text { (Retail Luggage) } \\
B\end{array}$ & $\begin{array}{c}\text { Data Set } 4 \\
\text { (Travel) } \\
B\end{array}$ \\
\hline $\begin{array}{l}\text { Affiliate-Affiliate } \\
\text { Display-Disnlay }\end{array}$ & $0.053^{* * *}$ & & $1.065^{* * *}$ & $0.182^{* * *}$ \\
\hline $\begin{array}{l}\text { Display-Display } \\
\text { Newsletter-Newsletter }\end{array}$ & $0.107^{* * *}$ & & & $0.367^{* * *}$ \\
\hline PriceComp-PriceComp & & & $0.265^{* * *}$ & $0.108^{* * *}$ \\
\hline Referrer-Referrer & 0.291 & $0.346^{* * *}$ & $0.561^{* * * *}$ & \\
\hline Retargeting-Retargeting & $0.155^{* * * *}$ & & $0.150^{* *}$ & $0.149^{* *}$ \\
\hline SEAGeneric-SEAGeneric & $0.236^{* * * *}$ & $0.188^{* * *}$ & $0.132^{* * *}$ & $0.188^{* * * *}$ \\
\hline SEABrand-SEABrand & $0.139^{* * * *}$ & $0.345^{* * *}$ & $0.504^{* * * *}$ & $0.347^{* * * *}$ \\
\hline SEOGeneric-SEOGeneric & $-0.354^{* * *}$ & $0.259^{* * * *}$ & $0.167^{* * *}$ & $0.126^{* *}$ \\
\hline SEOBrand-SEOBrand & $0.121^{* * * *}$ & $0.125^{* * *}$ & $0.299^{* * *}$ & $0.207^{* * * *}$ \\
\hline Social-Social & & $0.136^{* * *}$ & & \\
\hline TypeIn-TypeIn & $0.239^{* * * *}$ & $0.167^{* * * *}$ & $0.520^{* * *}$ & \\
\hline PriceComp-Retargeting & $1.809^{*}$ & & 0.231 & $1.261^{* * *}$ \\
\hline PriceComp-SEAGeneric & 0.078 & & -0.167 & $0.875^{* * *}$ \\
\hline Referrer-SEAGeneric & 0.164 & $0.438^{* *}$ & 0.270 & \\
\hline Referrer-SEABrand & $1.241^{* * * *}$ & $1.160^{* * *}$ & $1.830^{* * *}$ & \\
\hline Referrer-SEOBrand & $0.887^{* * * *}$ & $0.531^{*}$ & $1.649^{* *}$ & \\
\hline SEABrand-SEAGeneric & $0.326^{* *}$ & $0.726^{* * *}$ & $1.149^{* * *}$ & $0.288^{* *}$ \\
\hline SEABrand-SEOBrand & $0.242^{* * * *}$ & $0.418^{* * *}$ & $0.388^{*}$ & 0.112 \\
\hline SEAGeneric-Display & & & & \\
\hline SEAGeneric-PriceComp & $2.676^{* * * *}$ & & 0.224 & $-0.501^{*}$ \\
\hline SEAGeneric-Retargeting & $0.711^{* * * *}$ & & $0.461^{* * * *}$ & 0.521 \\
\hline SEAGeneric-SEOGeneric & 0.152 & $0.287^{* * *}$ & 0.084 & $0.451^{* * *}$ \\
\hline SEAGeneric-TypeIn & $0.622^{* * * *}$ & $1.023^{* * *}$ & $1.448^{* * *}$ & \\
\hline SEOBrand-SEABrand & $0.616^{* * *}$ & $0.413^{* * *}$ & $0.739^{* * *}$ & $0.639^{* * * *}$ \\
\hline SEOGeneric-SEAGeneric & 0.198 & $0.440^{* * *}$ & -0.057 & 0.195 \\
\hline SEOGeneric-SEOBrand & $0.682^{* * * *}$ & $0.845^{* * *}$ & $1.895^{* * *}$ & $0.773^{* * *}$ \\
\hline TypeIn-SEAGeneric & $0.247^{* *}$ & $0.453^{* * *}$ & $0.574^{* * * *}$ & \\
\hline TypeIn-SEOGeneric & 0.184 & $0.475^{* * *}$ & -0.284 & \\
\hline$N$ & $1,184,582$ & 862,114 & 405,343 & 600,873 \\
\hline Observations & $1,398,267$ & 964,836 & 461,108 & 792,345 \\
\hline Time at risk & $1,863,964$ & $1,306,432$ & 655,963 & $1,171,897$ \\
\hline Log likelihood & $-112,666.5$ & $-181,493.4$ & $-93,816.5$ & $-115,963.9$ \\
\hline AIC & $225,383.0$ & $363,024.8$ & $187,683.1$ & $231,967.9$ \\
\hline $\mathrm{BIC}$ & $225,686.7$ & $363,248.6$ & $187,959.1$ & $232,199.5$ \\
\hline$R^{2}(\mathrm{D})$ & 0.132 & 0.139 & 0.189 & 0.405 \\
\hline$R^{2}(\mathrm{PH})$ & 0.043 & 0.045 & 0.101 & 0.297 \\
\hline
\end{tabular}

Note. Display was omitted.

${ }^{*} p<.10 ; * * p .05 ; * * * p .01$.

based on full data sets (i.e., also including journeys with one click) reveals that a substantial number of browsing patterns are rather short, consisting of one or two clicks only, which emphasizes the need for advertisers to take single (present) clicks or short click sequences into account when optimizing their advertising activities. Furthermore, when the click patterns consist of more than one click, most of the identified user browsing traits are channel homogeneous, embracing our prior findings. Third, looking at samples that include journeys with two or more clicks, the majority of browsing patterns comprise only one or two different channels. Thus, users do not focus on one channel, but rather a limited set of one or two 
TABLE 5

Estimation Results: Full Table on Browsing Patterns With Three Clicks (Model 2b)

\begin{tabular}{|c|c|c|c|c|}
\hline Variable & $\begin{array}{c}\text { Data Set } 1 \\
\text { (Fashion High Priced) } \\
B\end{array}$ & $\begin{array}{c}\text { Data Set } 2 \\
\text { (Fashion Midlevel) } \\
B\end{array}$ & $\begin{array}{c}\text { Data Set } 3 \\
\text { (Retail Luggage) } \\
B\end{array}$ & $\begin{array}{c}\text { Data Set } 4 \\
\text { (Travel) } \\
B\end{array}$ \\
\hline Affiliate-Affiliate-Affiliate & 0.010 & & $1.188^{* * *}$ & $0.172^{* * *}$ \\
\hline Display-Display-Display & & & & \\
\hline Newsletter-Newsletter-Newsletter & $0.099^{* * *}$ & & & $0.391^{* * *}$ \\
\hline PriceComp-PriceComp-PriceComp & & & $0.241^{* * *}$ & $0.100^{* * *}$ \\
\hline Referrer-Referrer-Referrer & 0.206 & $0.228^{*}$ & $0.437^{* *}$ & \\
\hline Retargeting-Retargeting-Retargeting & 0.084 & & 0.145 & $0.140^{*}$ \\
\hline SEAGeneric-SEAGeneric-SEAGeneric & $0.217^{* * *}$ & $0.157^{* * *}$ & $0.084^{* * *}$ & $0.140^{* * * *}$ \\
\hline SEABrand-SEABrand-SEABrand & $0.115^{* * *}$ & $0.221^{* * *}$ & $0.483^{* * *}$ & $0.326^{* * *}$ \\
\hline SEOGeneric-SEOGeneric-SEOGeneric & $-0.216^{* *}$ & $0.250^{* * *}$ & $0.153^{* * *}$ & $0.295^{* * *}$ \\
\hline SEOBrand-SEOBrand-SEOBrand & $0.106^{* * *}$ & 0.037 & $0.300^{* * *}$ & $0.266^{* * *}$ \\
\hline Social-Social-Social & & $0.120^{* * *}$ & & \\
\hline TypeIn-TypeIn-TypeIn & $0.177^{* * * *}$ & 0.017 & $0.456^{* * *}$ & \\
\hline Affiliate-TypeIn-TypeIn & $0.734^{* * * *}$ & & $2.334^{* * *}$ & \\
\hline Newsletter-TypeIn-TypeIn & $0.432^{* * * *}$ & & & \\
\hline PriceComp-SEAGeneric-SEAGeneric & & & -0.010 & $0.696^{* *}$ \\
\hline Referrer-Affiliate-Affiliate & $0.819^{* * * *}$ & & & \\
\hline SEABrand-TypeIn-TypeIn & $0.651^{* * * *}$ & 0.325 & $1.582^{* * *}$ & \\
\hline SEAGeneric-PriceComp-PriceComp & & & -0.093 & $0.677^{*}$ \\
\hline SEAGeneric-Retargeting-Retargeting & $0.962^{* *}$ & & -0.328 & $1.755^{* *}$ \\
\hline SEAGeneric-SEAGeneric-PriceComp & $3.557^{* 2 * 3}$ & & $0.572^{* *}$ & -0.150 \\
\hline SEAGeneric-SEAGeneric-Retargeting & $0.936^{*}$ & & $0.803^{* * *}$ & -0.083 \\
\hline SEAGeneric-SEAGeneric-SEABrand & $0.778^{* * * *}$ & $0.843^{* * * *}$ & $1.648^{* * * *}$ & $1.341^{* * * *}$ \\
\hline SEAGeneric-SEAGeneric-SEOGeneric & 0.409 & $0.531^{* * * *}$ & $0.385^{* *}$ & $0.884^{* * * *}$ \\
\hline SEAGeneric-SEAGeneric-TypeIn & $0.412^{*}$ & $0.971^{* * *}$ & $1.718^{* * *}$ & \\
\hline SEAGeneric-SEOGeneric-SEAGeneric & $0.861^{* * *}$ & -0.138 & -0.332 & $0.880^{* * * *}$ \\
\hline SEAGeneric-SEOGeneric-SEOGeneric & -0.403 & 0.286 & $0.321^{*}$ & 0.339 \\
\hline SEAGeneric-Social-Social & & $0.699^{* * *}$ & & \\
\hline SEAGeneric-TypeIn-TypeIn & $0.489^{* * * *}$ & $0.467^{* * * *}$ & $1.266^{* * * *}$ & \\
\hline SEOBrand-TypeIn-TypeIn & $0.738^{* * *}$ & $0.782^{* * *}$ & 0.428 & \\
\hline SEOGeneric-SEAGeneric-SEOGeneric & 0.285 & $0.634^{* * *}$ & -0.128 & -0.182 \\
\hline SEOGeneric-SEOBrand-SEOBrand & $0.795^{* * *}$ & $0.993^{* * *}$ & $2.988^{* * *}$ & $1.134^{* * *}$ \\
\hline SEOGeneric-SEOGeneric-SEAGeneric & -0.664 & $0.550^{* * *}$ & 0.077 & $0.730^{* * *}$ \\
\hline SEOGeneric-Social-Social & & $0.789^{* * *}$ & & \\
\hline SEOGeneric-TypeIn-TypeIn & 0.354 & $0.465^{* * *}$ & 0.525 & \\
\hline TypeIn-Affiliate-Affiliate & $1.285^{* * *}$ & & 0.219 & \\
\hline TypeIn-Referrer-Affiliate & $1.514^{* * *}$ & & & \\
\hline TypeIn-Retargeting-Retargeting & $0.483^{*}$ & & $2.201^{* * *}$ & \\
\hline TypeIn-SEAGeneric-SEAGeneric & 0.072 & $0.624^{* * *}$ & $0.405^{*}$ & \\
\hline TypeIn-SEOGeneric-SEOGeneric & 0.260 & $0.357^{*}$ & -0.559 & \\
\hline TypeIn-Social-Social & & $0.903^{* * *}$ & & \\
\hline$N$ & $1,184,582$ & 862,114 & 405,343 & 600,873 \\
\hline Observations & $1,398,267$ & 964,836 & 461,108 & 792,345 \\
\hline Time at risk & $1,863,964$ & $1,306,432$ & 655,963 & $1,171,897$ \\
\hline Log likelihood & $-112,827.1$ & $-181,731.4$ & $-94,082.3$ & $-116,809.8$ \\
\hline AIC & $225,718.2$ & $363,510.9$ & $188,226.6$ & $233,659.6$ \\
\hline
\end{tabular}


TABLE 5

Estimation Results: Full Table on Browsing Patterns With Three Clicks (Model 2b) (Continued)

\begin{tabular}{lcccc}
\hline & $\begin{array}{c}\text { Data Set 1 } \\
\text { (Fashion High Priced) }\end{array}$ & $\begin{array}{c}\text { Data Set 2 } \\
\text { (Fashion Midlevel) }\end{array}$ & $\begin{array}{c}\text { Data Set 3 } \\
\text { (Retail Luggage) }\end{array}$ & $\begin{array}{c}\text { Data Set 4 } \\
\text { (Travel) }\end{array}$ \\
Variable & $B$ & $B$ & $B$ & $B$ \\
\hline BIC & $226,107.0$ & $363,793.6$ & $188,568.9$ & $233,891.2$ \\
$R^{2}(\mathrm{D})$ & 0.057 & 0.076 & 0.138 & 0.357 \\
$R^{2}(\mathrm{PH})$ & 0.024 & 0.028 & 0.062 & 0.206 \\
\hline
\end{tabular}

Note. Display was omitted.

$* p<.10 ; * * p<.05 ; * * * p<.01$.

TABLE 6

Estimation Results: Full Table on Browsing Patterns With Four Clicks (Model 2c)

\begin{tabular}{|c|c|c|c|c|}
\hline & $\begin{array}{c}\text { Data Set } 1 \\
\text { (Fashion High Priced) }\end{array}$ & $\begin{array}{c}\text { Data Set } 2 \\
\text { (Fashion Midlevel) }\end{array}$ & $\begin{array}{c}\text { Data Set } 3 \\
\text { (Retail Luggage) }\end{array}$ & $\begin{array}{c}\text { Data Set } 4 \\
\text { (Travel) }\end{array}$ \\
\hline Variable & $B$ & $B$ & $B$ & $B$ \\
\hline Affiliate-Affiliate-Affiliate-Affiliate & -0.003 & & $1.151^{* * *}$ & $0.158^{* * * *}$ \\
\hline Display-Display-Display-Display & & & & \\
\hline Newsletter-Newsletter-Newsletter-Newsletter & $0.088^{* * *}$ & & & $0.412^{* * * *}$ \\
\hline PriceComp-PriceComp-PriceComp-PriceComp & & & $0.266^{* * *}$ & $0.091^{* * * *}$ \\
\hline Referrer-Referrer-Referrer-Referrer & 0.165 & -0.159 & $0.437^{* *}$ & \\
\hline Retargeting-Retargeting-Retargeting-Retargeting & 0.104 & & 0.085 & $0.154^{*}$ \\
\hline $\begin{array}{l}\text { SEAGeneric-SEAGeneric-SEAGeneric- } \\
\text { SEAGeneric }\end{array}$ & $0.221^{* *}$ & 0.089 & $0.068^{* * *}$ & $0.158^{* * * *}$ \\
\hline SEABrand-SEABrand-SEABrand-SEABrand & $0.080^{* * * *}$ & $0.146^{* * * *}$ & $0.438^{* * * *}$ & $0.311^{* * * *}$ \\
\hline $\begin{array}{l}\text { SEOGeneric-SEOGeneric-SEOGeneric- } \\
\text { SEOGeneric }\end{array}$ & $-0.313^{*}$ & $0.237^{* * * *}$ & 0.053 & $0.286^{* * * *}$ \\
\hline $\begin{array}{l}\text { SEOBrand-SEOBrand-SEOBrand-SEOBrand } \\
\text { Social-Social-Social-Social }\end{array}$ & $0.111^{* * *}$ & $\begin{array}{r}-0.048 \\
0.109^{* * *}\end{array}$ & 0.199 & $0.298^{* * * *}$ \\
\hline TypeIn-TypeIn-TypeIn-TypeIn & $0.129^{* * *}$ & $-0.114^{* * *}$ & $0.365^{* * *}$ & \\
\hline Affiliate-TypeIn-TypeIn-TypeIn & $0.626^{* * *}$ & & $1.639^{* * *}$ & \\
\hline Newsletter-Newsletter-Newsletter-TypeIn & $0.757^{* * *}$ & & & \\
\hline PriceComp-PriceComp-PriceComp-SEAGeneric & & & -1.266 & $1.262^{* * *}$ \\
\hline Referrer-Social-Social-Social & & $0.956^{* * *}$ & & \\
\hline SEAGeneric-Affiliate-Affiliate-Affiliate & $1.481^{* * *}$ & & $2.018^{* * *}$ & $2.087^{* * * *}$ \\
\hline SEAGeneric-Affiliate-Affiliate-TypeIn & & & $2.582^{* *}$ & \\
\hline SEABrand-SEOBrand-SEOBrand-SEABrand & $0.532^{*}$ & -0.482 & $2.209^{* * *}$ & $1.489^{* * * *}$ \\
\hline SEABrand-SEOBrand-SEOBrand-SEOBrand & $0.847^{* * *}$ & $0.898^{* * *}$ & $2.583^{* * *}$ & $0.644^{* * * *}$ \\
\hline SEABrand-TypeIn-TypeIn-TypeIn & $0.582^{* *}$ & $0.916^{* * *}$ & $2.699^{* * *}$ & \\
\hline SEAGeneric-PriceComp-PriceComp-SEAGeneric & & & -0.438 & $1.632^{* *}$ \\
\hline SEAGeneric-Referrer-SEAGeneric-SEAGeneric & & $2.199^{* *}$ & & \\
\hline SEAGeneric-Retargeting-Retargeting-Retargeting & 1.133 & & 0.124 & $2.882^{* * * *}$ \\
\hline SEAGeneric-SEABrand-SEABrand-SEABrand & 0.125 & 0.085 & $0.840^{* * *}$ & $0.872^{\text {**** }}$ \\
\hline SEAGeneric-SEAGeneric-SEAGeneric-PriceComp & & & $0.677^{* *}$ & 0.028 \\
\hline SEAGeneric-SEAGeneric-SEAGeneric-Retargeting & $1.612^{* *}$ & & 0.388 & 1.401 \\
\hline SEAGeneric-SEAGeneric-SEAGeneric-SEABrand & 0.109 & $1.094^{* *}$ & $1.546^{* * * *}$ & $1.771^{* * * *}$ \\
\hline
\end{tabular}


TABLE 6

Estimation Results: Full Table on Browsing Patterns With Four Clicks (Model 2c) (Continued)

\begin{tabular}{|c|c|c|c|c|}
\hline & $\begin{array}{c}\text { Data Set } 1 \\
\text { (Fashion High Priced) }\end{array}$ & $\begin{array}{c}\text { Data Set } 2 \\
\text { (Fashion Midlevel) }\end{array}$ & $\begin{array}{c}\text { Data Set } 3 \\
\text { (Retail Luggage) }\end{array}$ & $\begin{array}{l}\text { Data Set } 4 \\
\text { (Travel) }\end{array}$ \\
\hline Variable & $B$ & $B$ & $B$ & $B$ \\
\hline SEAGeneric-SEAGeneric-SEAGeneric-TypeIn & 0.445 & $1.643^{* * *}$ & $2.059^{* * * *}$ & \\
\hline $\begin{array}{l}\text { SEAGeneric-SEAGeneric-SEOGeneric- } \\
\text { SEOGeneric }\end{array}$ & & -0.419 & 0.197 & $1.881^{* * * *}$ \\
\hline SEAGeneric-SEOBrand-SEOBrand-SEOBrand & 0.452 & $1.197^{* *}$ & 0.693 & $1.390^{* * * *}$ \\
\hline $\begin{array}{l}\text { SEAGeneric-SEOGeneric-SEAGeneric- } \\
\text { SEAGeneric }\end{array}$ & 0.200 & 0.188 & 0.302 & $1.275^{* * *}$ \\
\hline $\begin{array}{l}\text { SEAGeneric-SEOGeneric-SEOGeneric- } \\
\text { SEOGeneric }\end{array}$ & $0.899^{*}$ & 0.230 & $0.859^{* * * *}$ & -0.502 \\
\hline SEAGeneric-Social-Social-Social & & $0.707^{* * *}$ & & \\
\hline SEAGeneric-TypeIn-TypeIn-TypeIn & $0.416^{* *}$ & 0.225 & $1.717^{* * *}$ & \\
\hline SEOGeneric-Affiliate-Affiliate-Affiliate & $1.528^{* * * *}$ & & $3.612^{* * * *}$ & $3.930^{* * *}$ \\
\hline $\begin{array}{l}\text { SEOGeneric-SEAGeneric-SEOGeneric- } \\
\text { SEOGeneric }\end{array}$ & & $1.251^{* * *}$ & -0.602 & $0.859^{* *}$ \\
\hline SEOGeneric-SEOBrand-SEOGeneric-SEOGeneric & $0.896^{* * *}$ & -0.255 & & $1.583^{* * *}$ \\
\hline $\begin{array}{l}\text { SEOGeneric-SEOGeneric-SEOGeneric- } \\
\text { SEAGeneric }\end{array}$ & -1.090 & $0.634^{* *}$ & $0.443^{*}$ & $1.013^{* * *}$ \\
\hline SEOGeneric-SEOGeneric-SEOGeneric-SEOBrand & 0.470 & $1.169^{* * *}$ & 1.639 & $1.709^{* * *}$ \\
\hline SEOGeneric-SEOGeneric-SEOGeneric-TypeIn & -1.035 & $1.329^{* * *}$ & 1.012 & \\
\hline SEOGeneric-Social-Social-Social & & $0.720^{* * * *}$ & & \\
\hline SEOGeneric-TypeIn-TypeIn-TypeIn & -0.057 & $0.364^{* *}$ & 0.678 & \\
\hline Social-Social-Social-SEAGeneric & & $0.946^{* * *}$ & & \\
\hline Social-Social-Social-SEOGeneric & & $0.782^{* * * *}$ & & \\
\hline Social-Social-Social-TypeIn & & $0.775^{* * *}$ & & \\
\hline Social-TypeIn-TypeIn-TypeIn & & $0.533^{* * *}$ & & \\
\hline TypeIn-Affiliate-Affiliate-Affiliate & $1.846^{* * * *}$ & & 0.082 & \\
\hline TypeIn-Affiliate-Affiliate-TypeIn & 0.199 & & $2.445^{* *}$ & \\
\hline TypeIn-Retargeting-Retargeting-Retargeting & 0.103 & & $1.593^{* * *}$ & \\
\hline TypeIn-Social-Social-Social & & $0.475^{* * *}$ & & \\
\hline$N$ & $1,184,582$ & 862,114 & 405,343 & 600,873 \\
\hline Observations & $1,398,267$ & 964,836 & 461,108 & 792,345 \\
\hline Time at risk & $1,863,964$ & $1,306,432$ & 655,963 & $1,171,897$ \\
\hline Log likelihood & $-112,901.1$ & $-181,785.5$ & $-94,246.3$ & $-117,232.4$ \\
\hline AIC & $225,868.1$ & $363,637.0$ & $188,566.7$ & $234,518.8$ \\
\hline BIC & $226,269.1$ & $364,025.7$ & $188,975.2$ & $234,831.6$ \\
\hline$R^{2}(\mathrm{D})$ & 0.016 & 0.028 & 0.105 & 0.319 \\
\hline$R^{2}(\mathrm{PH})$ & 0.027 & 0.023 & 0.038 & 0.158 \\
\hline
\end{tabular}

Note. Display was omitted.

$* p<.10 ; * * p<.05 ; * * * p<.01$

channels tends to dominate their browsing traits. Due to the explorative nature of cluster analysis, these results do not serve to foresee conversion events.

Specific channel homogeneous effects (Model 2). Looking at the channel homogeneous click sequences, with the exception of one covariate (e.g., Model 2a, DS 1: SEOgenericSEOgeneric $b=-0.354, p<.01$ ), all significant effects show positive signs embracing our prior findings (e.g., Model 2a,
DS 3: Referrer-Referrer $b=0.561, p<.01)$. However, the magnitude of these effects, consistently across data sets, is surpassed by specific channel heterogeneous browsing patterns for each data set and model (e.g., Model 2a, DS 3: ReferrerSEAbranded $b=1.830, p<.01)$. Reflecting the exact sequential order of click patterns adds another level of detail, also reflecting the interplay of channels. Though these findings apply across industries, the comparison of channel 
homogeneous sequences of different length shows interesting dissimilarities. For the two fashion retailers, when comparing click sequences of two or more clicks the relative magnitude of the channel homogeneous effects appears stable in the context of their channel heterogeneous pendants. But the magnitude of these effects diminishes in the context of channel heterogeneous effects for the travel company. In other words, sequences of the identical channel seem less favorable for attracting customers to travel products. These variations may be moderated by the customer's price sensitivity and the complexity of the corresponding product category (Mehta, Rajiv, and Srinivasan 2003).

Turning to channel-related effects discloses further industry-specific and cross-industrial aspects, which remain comparably stable for channel homogeneous click sequences of from two to four clicks. For travel, sequences of newsletter, as well as branded paid and branded unpaid search, show strong positive effects toward conversion events (e.g., Model 2a, DS 4: Newsletter-Newsletter $b=0.367, p<.01$; SEAbrandedSEAbranded $b=0.347, p<.01$ ). While branded search contacts also express strong positive effects for retailers, it is notable that direct type-in, referrers, affiliates, and partly generic search sequential contacts become advantageous predictors for conversion events-which also mirrors our prior findings in Model 1. Particularly, affiliates and referrers for the luggage retailer, and referrers for one fashion retailer, indicate positive effects (Model 2a, DS2: Referrer-Referrer $b=0.346, p<.01$; DS3: Referrer-Referrer $b=0.561, p<.01$; Affiliate-Affiliate $b=1.065, p<.01$ ). Thus, for luggage products and midlevel fashion, both affiliates and referrers may serve as relevant gateways for catalyzing purchase events. The explanation may route back to the question of whether these channels initialize purchase paths or finalize purchase transactions, as they often include coupons that allow affiliate websites to "free-ride" on previous contacts, at least when leveraged by more advanced online users (Berman 2016). For higher-priced fashion products, both direct type-in and generic paid search represent the most promising channel homogeneous click sequences (Model 2a, DS1: TypeIn-TypeIn $b=0.239, p<.01$ ), potentially because more exclusive online stores may have invested more resources into their brand recognition. This makes branded searches less relevant, as their customer base may directly route to their website using the corresponding URL. Notably, these findings remain stable across click sequences of different length.

Specific channel heterogeneous effects. Before interpreting the effects of channel heterogeneous click sequences, a remarkable finding is that, independent from the length of the analyzed click sequence, the majority of browsing patterns cover only two different channels, strengthening our prior findings that user preferences are channel homogeneous rather than channel heterogeneous. Yet the interplay between two channels reveals amplifying effects that surpass the effect of pure channel homogeneous sequences.
Across all data sets and lengths of click sequences, branded exposures, including search as well as direct type-ins, play a pivotal role in preparing purchase transactions (Model 2a, DS1: Referrer-SEAbranded $b=1.241, p<.01$; DS2: Referrer-SEAbranded $b=1.160, p<.01$; DS3: SEOgeneric-SEObranded $b=1.895, p<.01$; DS4: Referrer-SEAbranded $b=$ $0.773, p<.01)$. However, branded contacts are most effective if preceded by other channels, such as referrers or generic search contacts (Yang and Ghose 2010), deepening our prior findings on search for Model 1. The positive effect levels off for sequences of sole branded customer interactions, likely because the transition from generic to branded contacts expresses progression along the path to purchase. This may be driven by the differing nature of apparent cost of generic and branded search contacts that generate positive spillovers to (subsequent) branded search contacts.

In addition, for online retailers, click sequences including referrers and, across all data sets, click sequences including affiliates, show strong positive effects on purchase propensity. While referrer contacts tend to be situated at the beginning or in the middle of click sequences, affiliates terminate click sequences (e.g., DS1, Model 2b: TypeIn-Referrer-Affiliate $b=1.541, p<.05$; Model 2c: SEAgeneric-Affiliate-Affiliate-Affiliate $b=1.481, p<.01)$. This supports the initial idea that affiliates may serve to finalize purchase events, in part, at the cost of potential advertiser revenues, as affiliates regularly include discounts (Berman 2016). Along these lines, affiliate contacts emerge in click sequences consisting of three or more clicks and play only a subordinate role in shorter click patterns. Thus, affiliates may still be necessary to successfully convince undecided users with longer journeys to purchase at the corresponding store-users that may procrastinate their purchase or be lost otherwise (Amir and Ariely 2001).

Other relevant channels in channel heterogeneous click sequences are price comparison websites, as well as retargeted display banners. Interestingly, these findings seem to be associated with the (relative) price points of the corresponding offerings rather than with the respective industry. Two-click sequences, including price comparisons, represent the strongest positive effect for the high-priced fashion retailer, as well as for the travel website (Model 2a, DS1: SEAgeneric-PriceComparison $b=1.809, p=.10$; DS4: PriceComparisonRetargeting $b=1.261, p=.05$ ). Looking at longer click sequences, price comparison contacts remain among the strongest positive predictors for the first fashion retailer and the travel company, and they gain importance for the luggage company (e.g., Model 2a, DS1: SEAgeneric-SEAgeneric-PriceComparison $b=3.557, p=.01$; DS3: SEAgeneric-SEAgeneric-PriceComparison $b=0.572, p=.05$ ). Thus, price comparisons seem less dependent on the product category than on the actual price level of the offering and, in consequence, on the price sensitivity of potential customers (Mehta, Rajiv, and Srinivasan 2003). Expectedly, the importance of retargeted display advertisements for anticipating conversion 
events grows with the length of click sequences, and is typically anteceded by other contacts, such as search for the fashion and travel companies or direct type-in for the luggage retailer.

As final channel, the interpretation of social media contacts reveals interesting usage patterns, although social media contacts occur only within one data set. First, social media contacts become relevant in longer click sequences that entail more than two clicks, and they are especially suitable as predictors whenever paired with other channels. While the positive effect of stand-alone social media sequences is limited, sequences dominated by social media contacts yet enriched by single clicks on other channels (e.g., search, referrers, or direct type-ins) become comparably strong (e.g., Model 2c, DS2: Social-Social-Social-SEAgeneric $b=0.946, p=.01)$. Consequently, users who react more than once to a distinct social media campaign seem to represent a promising customer group that should be taken into account by advertisers, especially those outside the sphere of social media.

\section{DISCUSSION}

This study develops a model for analyzing online journey data that aims to illuminate user channel preferences in a multichannel online environment and for focusing on user click patterns-both general channel contacts (Model 1) and specific channel click sequences (Model 2) across multiple industries. Most important, our research adds to advertising theory and practice in numerous ways.

First, we link user channel preferences and purchase decision making by analyzing present channel preferences and past channel stock and by introducing interactions between present and past channel exposures (Model 1). Across all data sets, we find that in a pure online environment users commonly select their preferred channels on the path to purchase: Present clicks on affiliates, branded paid and branded unpaid search, and social media, as well as (repeated) past clicks on retargeting, are positively associated with conversion events. Other channels are industry specific, or are affected by various underlying effects, such as price comparison websites that may be subject to price competitiveness or the degree of product commoditization. Combining past channel exposure with present clicks clearly establishes that customers who are prone to convert use a limited set, rather than a multitude, of channels. Notably, then, customer journeys that entail repeated use of a limited set of channels present a direct opportunity for developing successful online advertising strategies. In contrast, user journeys dominated by multichannel exposures are ineffective, contradicting studies that present the common view of multichannel exposures being effective (e.g., Chang and Thorson 2004). These conclusions add to prior multichannel research (Naik and Raman 2003; Tellis et al. 2005), extending established knowledge on synergetic channel effects (Li and Kannan 2014).
We also identify new insights on specific channel click sequences that reflect the exact browsing patterns of users who entail up to four subsequent clicks (Model 2). Our study verifies the consistent relevance of homogeneous channel sequences across data sets derived from our previous Model 1, thereby mirroring channel user preferences-a consistent relevance that is also shown in multichannel relational communication (Godfrey, Seiders, and Voss 2011). It is worthwhile for online advertisers to note that the effect of channel homogeneous click sequences remains relatively stable when extending the length of the click sequences (from two to four clicks) for retail (and especially for fashion retail), though the positive effect levels off for travel. The different outcomes for travel may link to the complexity of travel products, which often require more complex browsing paths to finalize purchase decisions. In addition to the relevance of idiosyncratic channel preferences, we reveal that click sequences of up to four clicks that also include two channels tend to surpass the effects of pure channel homogeneous click sequences of the same length. Importantly, these click sequences seldom include more than two channels, confirming our previous claim regarding the effectiveness of limited channel use by potential customers. Across industries, we find that click sequences, including branded contacts such as search or direct type-in, are positively related to conversions. Retailer click sequences, including affiliates and referrers, show strong positive effects on conversions. However, referrer appears to indicate initialized browsing sequences, while affiliate is seen when users are prone to conclude a purchase decision. This distinction may arise because affiliate websites often include coupons to catalyze purchase decisions, though at the expense of potential revenues (Berman 2016). In contrast, our results show that price comparison is less industry specific and is associated with user price sensitivity (Mehta, Rajiv, and Srinivasan 2003), such that effects for the high-priced fashion retailer appear similar to the effects for the travel company. Thus, our study deepens research on the introduction of holistic marketing impact models that build on individual-level customer path data (Hui, Fader, and Bradlow 2009).

Third, our research also links theory to practice-focusing on theoretical approaches to advertising effectiveness and to clickstream analysis (Little 2004; Yadav and Pavlou 2014). The opportunity to derive practical generalizations and industry-specific conclusions in our study arose from our investigation of four real-world data sets from three industries that comprise the complete range of channels that can be applied (Li and Kannan 2014). Results from our application of cluster analysis confirm that users who are likely to purchase do, indeed, prefer a limited set of channels rather than myriad online vehicles. These findings contain valuable implications for practitioners in shaping their online strategies. The generalized findings may translate directly into concrete advertising applications. Industry-specific insights provide advertisers with methods for tracking the browsing histories of individual 
users and applying those observations to online strategies. Another aspect of our study that extends to practice is our focus on retargeting techniques-both in the context of methodology and of the study results. Approximating our approach to conversion likelihood and individual customer value is applicable, particularly, in developing strategies for real-time bidding. Furthermore, our insights not only are relevant for targeting current customers but also are effectively applied to new users identified from their web browsing. Appropriate retargeting and budget allocation aimed toward well-selected channels have been shown to improve advertising effectiveness (Lambrecht and Tucker 2013; Tucker 2013). These examples illustrate that a range of insights derived from our results can be used to improve advertising effectiveness.

\section{LIMITATIONS AND OUTLOOK}

Our research is subject to several limitations that may serve to stimulate future research. One area that deserves further investigation is related to cookie-tracking technology, and specifically to tracking limitations that result from cookie deletion or multidevice usage. Although cookie tracking remains an industry standard for clickstream analyses (Tucker 2013), new tracking technologies may mitigate tracking limitations, but the same new technology calls for new research. Furthermore, our data sets comprise clickstreams that omit impression data so that we do not track effects for users who only view an advertisement. Studies that involve impressions as well as clicks (e.g., in a two-stage model) could be a useful extension of the present study.

Moreover, while our analysis of real-world field data measures direct customer-advertiser interactions, user motivation and (potential) psychological mechanisms remain masked. The data thus enable us to measure correlation, which is incongruent with causality. Another useful avenue for future research is continuing our research on the association of purchase inclination and channel/channel category exposure by developing substantive field experiments (ideally in combination with survey-based studies).

Although we applied a set of cluster analyses to thoroughly mine all data sets for hidden browsing paths, it is possible that we missed relevant patterns. We note that limiting channel combinations was the appropriate choice for our study, as a fully comprehensive analysis that included all possible channel combinations would have created an unwieldy framework from which to create our models. However, practical future studies on the interplay of channels in multichannel settings might confront the potential complexities by selecting an investigatory model that can support a more complex number of variables to extend our originating research. Therefore, a data-driven approach to clustering online channels-ideal in this era of big data-may further enhance the predictive power for innovative online strategies.

\section{SUPPLEMENTAL DATA}

Supplemental data for this article can be accessed at www.tandfonline.com/ujoa.

\section{REFERENCES}

Agarwal, Ashish, Kartik Hosanagar, and Michael D. Smith (2011), "Location, Location, Location: An Analysis of Profitability of Position in Online Advertising Markets," Journal of Marketing Research, 48 (6), 1057-73.

Amir, On, and Dan Ariely (2001), "The Pain of Deciding: Indecision, Procrastination, and Consumer Choice Online," working paper, https://pdfs. semanticscholar.org/8620/be036cd976ab8a99397fcfa7502799765f01.pdf.

Bellman, Richard E. (1961), Adaptive Control Processes: A Guided Tour, Princeton, NJ: Princeton University Press.

Berman, Ron (2016), "Beyond the Last Touch: Attribution in Online Advertising," available https://ssrn.com/abstract=2384211 or http://dx.doi. org/10.2139/ssrn.2384211.

Braun, Michael, and Wendy W. Moe (2013), "Online Display Advertising: Modeling the Effects of Multiple Creatives and Individual Impression Histories," Marketing Science, 32 (5), 753-67.

Breuer, Ralph, Malte Brettel, and Andreas Engelen (2011), "Incorporating Long-Term Effects in Determining the Effectiveness of Different Types of Online Advertising," Marketing Letters, 22 (4), 327-40.

Broder, Andrei (2002), “A Taxonomy of Web Search,” ACM SIGIR Forum, 36 (2), 3-10.

Calinski, T., and J. Harabasz (1974), "A Dendrite Method for Cluster Analysis," Communications in Statistics: Theory and Methods, 3 (1), 1-27.

Chang, Yuhmiin, and Esther Thorson (2004), "Television and Web Advertising Synergies," Journal of Advertising, 33 (2), 75-84.

Chatterjee, Patrali, Donna L. Hoffman, and Thomas P. Novak (2003), "Modeling the Clickstream: Implications for Web-Based Advertising Efforts," Marketing Science, 22 (4), 520-41.

Cox, David Roxbee (1972), "Regression Models and Life-Tables," Journal of the Royal Statistical Society, Series B, 34 (2), 187-220.

Danaher, Peter J., and Tracey S. Dagger (2013), "Comparing the Relative Effectiveness of Advertising Channels: A Case Study of a Multimedia Blitz Campaign," Journal of Marketing Research, 50 (4), 517-34.

Flosi, Stephanie, Gian M. Fulgoni, and Andrea Vollman (2013), "If an Advertisement Runs Online and No One Sees It, Is It Still an Ad? Empirical Generalizations in Digital Advertising," Journal of Advertising Research, 53 (2), 192-99.

Fulgoni, Gian M., and Marie Pauline Mörn (2009), "Whither the Click? How Online Advertising Works," Journal of Advertising Research, 49 (2), 134-42.

Ghose, Anindya, and Sha Yang (2009), "An Empirical Analysis of Search Engine Advertising: Sponsored Search in Electronic Markets," Management Science, 55 (10), 1605-22.

Godfrey, Andrea, Kathleen Seiders, and Glenn B. Voss (2011), "Enough Is Enough! The Fine Line in Executing Multichannel Relational Communication," Journal of Marketing, 75 (4), 94-109.

Hubert, L. J., and P. Arabie (1985), “Comparing Partitions,” Journal of Classification, 2, 193-218.

Hui, Sam K., Peter S. Fader, and Eric T. Bradlow (2009), "Path Data in Marketing: An Integrative Framework and Prospectus for Model Building," Marketing Science, 28 (2), 320-35.

Jansen, Bernard J., Kate Sobel, and Mimi Zhang (2011), "The Brand Effect of Key Phrases and Advertisements in Sponsored Search," International Journal of Electronic Commerce, 16 (1), 77-106.

Jerath, Kinshuk, Liye Ma, and Young-Hoon Park (2014), "Consumer Click Behavior at a Search Engine: The Role of Keyword Popularity," Journal of Marketing Research, 51 (4), 480-86.

Kireyev, Pavel, Koen Pauwels, and Sunil Gupta (2013), "Do Display Ads Influence Search? Attribution and Dynamics in Online Advertising," working paper 13-070, Harvard Business School, Boston, MA, February, http:// www.hbs.edu/faculty/PublicationFiles/13-070.pdf. 
Klapdor, Sebastian, Eva Anderl, Jan Schumann, and Florian Von Wangenheim (2015), "Using Multichannel Behavior to Predict Online Conversions," Journal of Advertising Research, 55 (4), 433-42.

- Florian von Wangenheim, and Jan Schumann (2014), "Finding the Right Words: The Influence of Linguistic-, Content-, and User-Related Keyword Characteristics on Performance of Paid Search Campaigns," Journal of Interactive Marketing, 28 (4), 285-301.

Lambrecht, Anja, and Catherine Tucker (2013), "When Does Retargeting Work? Information Specificity in Online Advertising," Journal of Marketing Research, 50 (5), 561-76.

LeClerc, France, Bern H. Schmitt, and Laurette Dubé (1995), "Waiting Time and Decision Making: Is Time Like Money?," Journal of Consumer Research, 22 (1), 110-19.

Li, Hongshuang Alice, and P. K. Kannan (2014), "Attributing Conversions in a Multichannel Online Marketing Environment: An Empirical Model and a Field Experiment," Journal of Marketing Research, 51 (1), 40-56.

Little, John D.C. (2004), "Comments on 'Models and Managers: The Concept of a Decision Calculus,"” Management Science, 50 (12 Suppl.), 1854-60.

MacInnis, Deborah J., and Bernard J. Jaworski (1989), "Information Processing from Advertisements: Toward an Integrative Framework," Journal of Marketing, 53 (4), 1-23.

Mehta, Nitin, Surendra Rajiv, and Kannan Srinivasan (2003), "Price Uncertainty and Consumer Search: A Structural Model of Consideration Set Formation," Marketing Science, 22 (1), 58-84.

Milligan, G. W., and M. Cooper (1985), "An Examination of Procedures for Determining the Number of Clusters in a Data Set," Psychometrika, 50 (2), 159-79.

Moe, Wendy W. (2003), "Buying, Searching, or Browsing: Differentiating between Online Shoppers Using In-Store Navigational Clickstream," Journal of Consumer Psychology, 13 (1), 29-39.

, and Peter S. Fader (2004a), "Capturing Evolving Visit Behavior in Clickstream Data," Journal of Interactive Marketing, 18 (1), 5-19.

— and (2004b), "Dynamic Conversion Behavior at E-Commerce Sites," Management Science, 50 (3), 326-35.

Mooi, E., and M. Sarstedt (2011), "Understanding cluster-analysis," in A Concise Guide to Market Research: The process, data, and methods using IBM SPSS statistics, E. Mooi and M. Sarstedt, eds., Heidelberg Dordrecht: Springer, pp. 259-83.

Montgomery, Alan L., Shibo Li, Kannan Srinivasan, and John C. Liechty (2004), "Modeling Online Browsing and Path Analysis Using Clickstream Data," Marketing Science, 23 (4), 579-95.

Naik, Prasad A., and Kalyan Raman (2003), "Understanding the Impact of Synergy in Multimedia Communications," Journal of Marketing Research, 40 (4), 375-88.
Qiu, Dingxi, and Edward C. Malthouse (2009), "Quantifying the Indirect Effects of a Marketing Contact," Expert Systems with Applications, 36 (3), 6446-52.

Rutz, Oliver J., and Randolph E. Bucklin (2011), "From Generic to Branded: A Model of Spillover in Paid Search Advertising," Journal of Marketing Research, 48 (1), 87-102. and Garrett P. Sonnier (2012), “A Latent Instrumental Variables Approach to Modeling Keyword Conversion in Paid Search Advertising," Journal of Marketing Research, 49 (3), 306-19.

-, Michael Trusov, and Randolph E. Bucklin (2011), "Modeling Indirect Effects of Paid Search Advertising: Which Keywords Lead to More Future Visits?," Marketing Science, 30 (4), 646-65.

Shankar, Venkatesh, and Edward C. Malthouse (2007), "The Growth of Interactions and Dialogs in Interactive Marketing," Journal of Interactive Marketing, 21 (2), 2-4.

Sismeiro, Catarina, and Randolph E. Bucklin (2004), "Modeling Purchase Behavior at an E-Commerce Web Site: A Task Completion Approach," Journal of Marketing Research, 41 (3), 306-23.

Steinley, Douglas, and Michael J. Brusco (2007), "Initializing $K$-Means Batch Clustering: A Critical Evaluation of Several Techniques," Journal of Classification, 24 (1), 99-121.

Tellis, Gerard J., Rajesh K. Chandy, Deborah MacInnis, and Pattana Thaivanich (2005), "Modeling the Microeffects of Television Advertising: Which Ad Works, When, Where, for How Long, and Why?," Marketing Science, 24 (3), 351-66.

_ and Philip Hans Franses (2006), "Optimal Data Interval for Estimating Advertising Response," Marketing Science, 25 (3), 217-29.

Tucker, Catherine (2013), "The Implications of Improved Attribution and Measurability for Antitrust and Privacy in Online Advertising Markets," George Mason Law Review, 20 (4), 1025-54.

Ward, J. H. (1963), "Hierarchical Grouping to Optimize an Objective Function," Journal of the American Statistical Association, 58 (301), 236-44

Wiesel, Thorsten, Koen Pauwels, and Joep Arts (2011), "Marketing's Profit Impact: Quantifying Online and Off-Line Funnel Progression," Marketing Science, 30 (4), 604-11.

Xia, Lan, and D. Sudharshan (2002), "Effects of Interruptions on Consumer Online Decision Processes," Journal of Consumer Psychology, 12 (3), 265-80.

Xu, Lizhen, Jason A. Duan, and Andrew Whinston (2014), "Path to Purchase: A Mutually Exciting Point Process Model for Online Advertising and Conversion," Management Science, 60 (6), 1392-1412.

Yadav, Manjit S., and Paul A. Pavlou (2014), "Marketing in Computer-Mediated Environments: Research Synthesis and New Directions," Journal of Marketing, 78 (1), 20-40.

Yang, Sha, and Anindya Ghose (2010), "Analyzing the Relationship between Organic and Sponsored Search Advertising: Positive, Negative, or Zero Interdependence?," Marketing Science, 29 (4), 602-23. 